\title{
Scattering of two and three physical pions at maximal isospin from lattice QCD
}

\author{
Extended Twisted Mass Collaboration
}

\author{
Matthias Fischer ${ }^{1,2}$, Bartosz Kostrzewa ${ }^{3}$, Liuming Liu ${ }^{4,5}$, Fernando Romero-López $^{6, \mathrm{a}} \mathbb{C}$, Martin Ueding ${ }^{1,2}$, \\ Carsten Urbach ${ }^{1,2}$ \\ ${ }^{1}$ Helmholtz-Institut für Strahlen- und Kernphysik, University of Bonn, 53115 Bonn, Germany \\ ${ }^{2}$ Bethe Center for Theoretical Physics, University of Bonn, 53115 Bonn, Germany \\ ${ }^{3}$ High Performance Computing and Analytics Lab, University of Bonn, 53115 Bonn, Germany \\ ${ }^{4}$ Institute of Modern Physics, Chinese Academy of Sciences, Lanzhou 730000, China \\ ${ }^{5}$ University of Chinese Academy of Sciences, Beijing 100049, China \\ ${ }^{6}$ Instituto de Física Corpuscular, Universitat de València and CSIC, 46980 Paterna, Spain
}

Received: 18 March 2021 / Accepted: 2 May 2021 / Published online: 20 May 2021

(C) The Author(s) 2021

\begin{abstract}
We present the first direct $N_{f}=2$ lattice QCD computation of two- and three- $\pi^{+}$scattering quantities that includes an ensemble at the physical point. We study the quark mass dependence of the two-pion phase shift, and the three-particle interaction parameters. We also compare to phenomenology and chiral perturbation theory (ChPT). In the two-particle sector, we observe good agreement to the phenomenological fits in $s$ - and $d$-wave, and obtain $M_{\pi} a_{0}=-0.0481(86)$ at the physical point from a direct computation. In the three-particle sector, we observe reasonable agreement at threshold to the leading order chiral expansion, i.e. a mildly attractive three-particle contact term. In contrast, we observe that the energy-dependent part of the three-particle quasilocal scattering quantity is not well described by leading order ChPT.
\end{abstract}

\section{Introduction}

Quantum chromodynamics (QCD) describes the interaction of quarks and gluons, while only hadrons (mesons and baryons) are experimentally observable. They are low energy bound states, or resonances of the former fundamental particles. Understanding the interactions of two or more hadrons is highly relevant for several reasons. For instance, resonances become visible only when studying the interaction of other hadrons. And for understanding experimental signatures of particle decays, the interactions of the final states need to be understood.

a e-mail: fernando.romero@uv.es (corresponding author)
Lattice QCD, the formulation of QCD on a spacetime lattice, offers the opportunity of first principles, numerical explorations of few-particle scattering amplitudes. Maybe the most obvious example for the importance of three-particle interactions is the $\omega$-meson, which decays predominantly into three pions with $J^{P}=1^{-}$[1]. Another one would be the Roper resonance [2], with both $N \pi$ and $N \pi \pi$ decay channels. However, since the investigation of three-particle interactions from lattice QCD is in its infancy, three weakly interacting pions with isospin $I=3$ is an interesting and important benchmark system.

The extraction of two-particle scattering amplitudes in Lattice QCD is by now well established for $2 \rightarrow 2$ systems, both theoretically [4-16], and in practice [3,17-40] (see Ref. [41] for a review). One of the most studied systems is isospin$2 \pi \pi$ scattering. To illustrate the state-of-the-art, we show in Fig. 1 the $\pi \pi I=2$ scattering length $M_{\pi} a_{0}$ as a function of $M_{\pi} / f_{\pi}$ comparing this work's result to the $N_{f}=2+1+1$ results of Ref. [3]. The new $N_{f}=2$ point at a slightly less than physical value of $M_{\pi} / f_{\pi}$ as well as the other two new points are compatible within errors with leading order (LO) ChPT (dashed line).

Over the last few years, theoretical and numerical work investigating three-particle scattering amplitudes from lattice QCD emerged as a hot topic. The finite-volume formalism exists following three different approaches: (i) generic relativistic effective field theory (RFT) [42-53], (ii) nonrelativistic effective field theory (NREFT) [54-59], and (iii) (relativistic) finite volume unitarity (FVU) [60,61] (see also Refs. [62-64] and Ref. [65] for a review). Lattice data [66-68] has been confronted with both the FVU $[61,67,68]$ and RFT [69] 


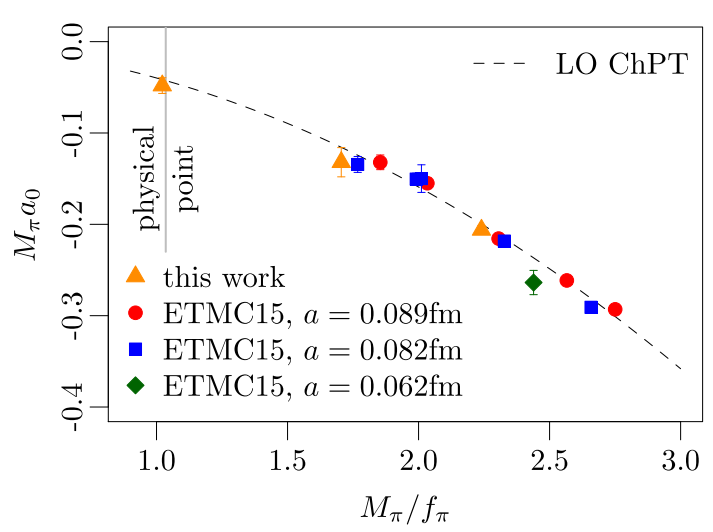

Fig. $1 I=2$ scattering length $M_{\pi} a_{0}$ as a function of $M_{\pi} / f_{\pi}$ comparing the $N_{f}=2+1+1$ ETMC twisted mass results [3] with this work. The dashed line represents the leading order ChPT prediction

formalisms (see also [70,71]). For a related approach see also Refs. [72-75].

In this article, we present results for scattering quantities of two and three-pion systems with maximal isospin, including for the first time an ensemble at the physical point. This work breaks new ground on several fronts: the first direct computation at the physical point of the $I=2 s$ - and $d$ wave phase shift, and the chiral dependence of the three- $\pi^{+}$ quasilocal interaction.

\section{Scattering amplitudes from lattice QCD}

The calculation of scattering amplitudes from lattice simulations proceeds in an indirect way. The required physical quantities from the lattice are the finite-volume interacting energies of two and three particles - the finite volume spectrum. The mapping between the finite volume spectrum and infinite-volume scattering quantities - the so-called quantization condition - is known but highly nontrivial. It is valid up to effects that vanish exponentially with the pion mass, $\sim \exp \left(-M_{\pi} L\right)$.

The two-particle quantization condition $(\mathrm{QC} 2)$ takes the form of a determinant equation [4-6] (we assume two identical scalars):

$\operatorname{det}\left[F_{2}^{-1}\left(\mathbf{P}, E^{*}, L\right)+\mathcal{K}_{2}\left(E^{*}\right)\right]=0$.

Here, $F_{2}$ and $\mathcal{K}_{2}$ are both matrices in angular momentum space $\ell, m$. The matrix elements of $F_{2}$ are kinematical functions (Lüscher zeta function) that depend on the three-momentum of the system, $\mathbf{P}$ and the center-of-mass $(\mathrm{CM})$ energy, $E^{*} .\left(\mathcal{K}_{2}\right)_{\ell m, \ell^{\prime} m^{\prime}}=\delta_{\ell m, \ell^{\prime} m^{\prime}}\left(\mathcal{K}_{2}\right)_{\ell}$ is simply the infinite-volume scattering K-matrix projected to the corresponding partial wave. In order to render the matrices finitedimensional, a truncation must be applied in $\ell, \ell^{\prime}$ by assum- ing that $\mathcal{K}_{2}$ vanishes for higher partial waves. Furthermore, the relations between $\mathcal{K}_{2}$, the phase shift $\left(\delta_{\ell}\right)$, and the scattering amplitude $\left(\mathcal{M}_{2}\right)$ are trivial. More details can be found in Ref. [41].

The three-particle quantization condition (QC3) for identical (pseudo)scalars in the RFT approach reads (G-parity is assumed) [42]:

$\operatorname{det}\left[F_{3}^{-1}(E, \mathbf{P}, L)+\mathcal{K}_{3, \mathrm{df}}\left(E^{*}\right)\right]=0$.

Even though this looks formally identical to Eq. 1, there are some distinct features. First, the matrices in Eq. 2 live in a larger $k \ell m$ space, where $\ell, m$ are the angular momentum indices of the interacting pair, and $k$ labels the threemomentum of the third particle - the spectator. Next, $F_{3}$ depends on geometric functions (like $F_{2}$ itself), but also on $\mathcal{K}_{2}$. Thus, two-particle interactions are a necessary ingredient for three-particle scattering. Note that an analytical continuation of $\mathcal{K}_{2}$ below threshold is needed for the QC3. Finally, $\mathcal{K}_{3 \text {,df }}$ is a real, singularity-free, quasilocal, intermediate three-particle scattering quantity - which we aim to determine. As in the case of the QC2, Eq. 2 is infinite-dimensional, and must be truncated. The truncation in $k$ is due to a cut-off function, whereas for $\ell, m$ one assumes that $\mathcal{K}_{3, \mathrm{df}}$ vanishes above some value of $\ell$, see Refs. [42,65] for details. Establishing the connection between $\mathcal{K}_{3 \text {,df }}$ and the physical scattering amplitude, $\mathcal{M}_{3}$ requires a set of integral equations, derived in Ref. [43] and solved in Ref. [47]. In this work, we focus only on the extraction of $\mathcal{K}_{3 \text {,df }}$.

In a finite volume, partial waves mix and, thus, $F_{2}$ and $F_{3}$ are nondiagonal in $\ell, m$. The correct labels are then irreducible representations (irreps) of the discrete symmetry group, which we label as $\Gamma$. The subduction of angular momenta into irreps is known [76, Table 2]. Therefore, one block-diagonalizes the quantization conditions into irreps, see Refs. [16,49,58,69].

\section{Lattice computation}

This work uses $N_{\mathrm{f}}=2$ flavour lattice QCD ensembles generated by the Extended Twisted Mass collaboration (ETMC) [80], including one ensemble at the physical pion mass see Table 1. For the ensemble generation the Iwasaki gauge action [81] was used together with Wilson clover twisted mass fermions at maximal twist [82]. The latter guarantees scaling towards the continuum with only $O\left(a^{2}\right)$ artefacts in the lattice spacing $a$ [83]. The presence of the clover term (with coefficient $c_{\mathrm{sw}}$ ) has been shown to further reduce the $O\left(a^{2}\right)$ artefacts, in particular isospin-breaking effects of the twisted-mass formulation, which have been empirically found to be very small for masses and decay constants [80]. 
Table $1 N_{f}=2$ Ensembles used in this work. The lattice spacing is $a=0.0914(15) \mathrm{fm}$, and $c_{\mathrm{SW}}=1.57551$. For the decay constant we use the normalization $f_{\pi}=\sqrt{2} F_{\pi} . M_{\pi} / f_{\pi}$ has been corrected for finite-size effects according to Refs. [77-79]

\begin{tabular}{|c|c|c|c|c|c|}
\hline Ensemble & $L^{3} \times T$ & $M_{\pi} / \mathrm{eV}$ & $a M_{\pi}$ & $M_{\pi} / f_{\pi}$ & \# confs. \\
\hline cA2.60.32 & $32^{3} \times 64$ & 340 & $0.1578(1)$ & $2.235(6)$ & 337 \\
\hline cA2.30.48 & $48^{3} \times 96$ & 242 & $0.11199(4)$ & $1.705(1)$ & 1403 \\
\hline cA2.09.48 & $48^{3} \times 96$ & 134 & $0.06205(4)$ & $1.022(1)$ & 1604 \\
\hline
\end{tabular}

For the two-pion scattering length with $I=2$, discretisation artefacts are only of order $O\left(a m_{q}\right)^{2}$, with $m_{q}$ the up/down quark mass [84]. Another possible source of $O\left(a^{2}\right)$ effects that should be mentioned is the $\pi^{0}$ contamination in the correlation functions due to the breaking of parity in twisted mass. However, it is also important to realise that at maximal isospin there is no mixing with other flavour states due to broken isospin symmetry. Parametrically, $O\left(a^{2}\right)$ artefacts are $\sim 2.5 \%$ and $O\left(a m_{q}\right)^{2} \leq 0.4 \%$ for this lattice spacing, and thus well below our statistical uncertainty.

The two- and three- $\pi^{+}$energy spectrum is measured from Euclidean correlation functions of operators with the corresponding quantum numbers. By means of the single pion operators $\left(\pi^{+}=-\overline{\mathrm{u}} \gamma_{5} \mathrm{~d}\right)$, we construct two-particle operators as

$\mathcal{O}_{\pi \pi}\left(p_{1}, p_{2}\right)=\sum_{x, y} e^{i p_{1} x+i p_{2} y} \pi^{+}(x) \pi^{+}(y)$,

where $p_{i}$ labels the momentum of each single pion, and similarly for three pions

$$
\begin{aligned}
\mathcal{O}_{\pi \pi \pi}\left(p_{1}, p_{2}, p_{3}\right)= & \sum_{x, y, z} e^{i p_{1} x+i p_{2} y+i p_{3} z} \\
& \times \pi^{+}(x) \pi^{+}(y) \pi^{+}(z) .
\end{aligned}
$$

Correlation functions are computed using the stochastic Laplacian-Heaviside smearing $[85,86]$ with algorithmic parameters as in Ref. [87]. In addition, operators that transform under a specific irrep of a discrete symmetry group are constructed following Ref. [32]. In the two-pion case we use the irreps $A_{1}^{(+)}, E^{(+)}, B_{1}$ and $B_{2}$, in the three pion channel $A_{1}^{(-)}, E^{(-)}, A_{2}, B_{1}$ and $B_{2}$, for all $\mathbf{P}^{2} \leq 4$ with $\mathbf{P}$ the centreof-mass momentum. We refer to Table 9 in the appendix for an overview. We extract the spectrum in each irrep independently using the generalized eigenvalue method (GEVM) $[6,88,89]$ and also the GEVM/PGEVM method [90], see the appendix for more details.

A technical issue of lattice calculations with (anti)periodic boundary conditions in the time direction is the presence of so-called thermal states, i.e. effects from states that propagate backwards in time across the boundary. They vanish with $M_{\pi} T \rightarrow \infty$, but at finite values of $T$, these effects are significant and need to be treated accordingly. In fact, thermal pollutions are one of the major systematic uncertainties in our calculation. We deal with them as follows: using the operators discussed above we build correlator matrices which are input to the GEVM/PGEVM which in turn have so-called principal correlators as output. From the latter energy levels and corresponding error estimates are extracted from bootstrapped, fully correlated fits to the data with fit ranges chosen by eye. We use five different treatments to arrive from a correlator matrix at an energy level. Details of those five treatments are explained in Appendix A1.

As also explained in Appendix A1, the different energy levels per principal correlator (up to five) are then combined using a correlated weighted average. However, to account for the spread between the different methods we use a procedure discussed in Ref. [32] to widen the resampling distribution: for energy level $E$ we compute the scaling factor

$w=\sqrt{\frac{(\delta E)^{2}+\sum_{Y}\left(\Delta E_{Y}\right)^{2}}{(\delta E)^{2}}}$,

where $\delta E$ is the statistical uncertainty of the weighted average and $\Delta E_{Y}$ is the difference between method $Y$ and the weighted average. By scaling the resampling distribution of the weighted average with $w$, we obtain a distribution that reflects both the statistical and the systematic uncertainties, while still being usable in the bootstrap analysis chain. The energy levels are publicly available [91].

The finite-volume scattering formalism is applicable under the assumption that exponential finite volume effects are negligible. On the physical point ensemble, we have $M_{\pi} L \approx 3$, which implies $e^{-M_{\pi} L} \sim 5 \%$ and might be considered to be at the edge of feasibility. However, based on a ChPT analysis, finite-volume effects are also proportional to $\left[M_{\pi} /\left(4 \pi F_{\pi}\right)\right]^{2}$, which at the physical point reduces finitevolume effects sizably. Moreover, as argued in Ref. [74], if the volume-dependent mass is used to analyze the multiparticle energy levels, the leading finite-size effects cancel. For the other two ensembles we have $M_{\pi} L>5$, which is safe concerning finite volume effects.

\section{Results}

In the case of two pions, by keeping only $s$-wave interactions in $A_{1}$ irreps, the projected QC2 becomes a one-to-one corre- 
Table $2 s$-wave fit results for the various ensembles using Eq. 6 with fixed $z_{2}^{2}=M_{\pi}^{2}$. Here we use only the two-pion levels in the $A_{1}^{+}$and $A_{1}$ irreps

\begin{tabular}{lllll}
\hline & $1 / B_{0}=M_{\pi} a_{0}$ & $B_{1}$ & $B_{2}$ & $\chi^{2} / \mathrm{dof}$ \\
\hline cA2.60.32 & $-0.2090(54)$ & $-2.3(3)$ & - & $19.06 /(16-2)$ \\
cA2.60.32 & $-0.2110(57)$ & $-3.1(6)$ & $0.4(2)$ & $15.96 /(16-3)$ \\
cA2.30.48 & $-0.132(16)$ & $-1.4(5)$ & - & $27.15 /(16-2)$ \\
cA2.09.48 & $-0.0477(90)$ & $-1.4(1.2)$ & - & $11.08 /(10-2)$ \\
\hline
\end{tabular}

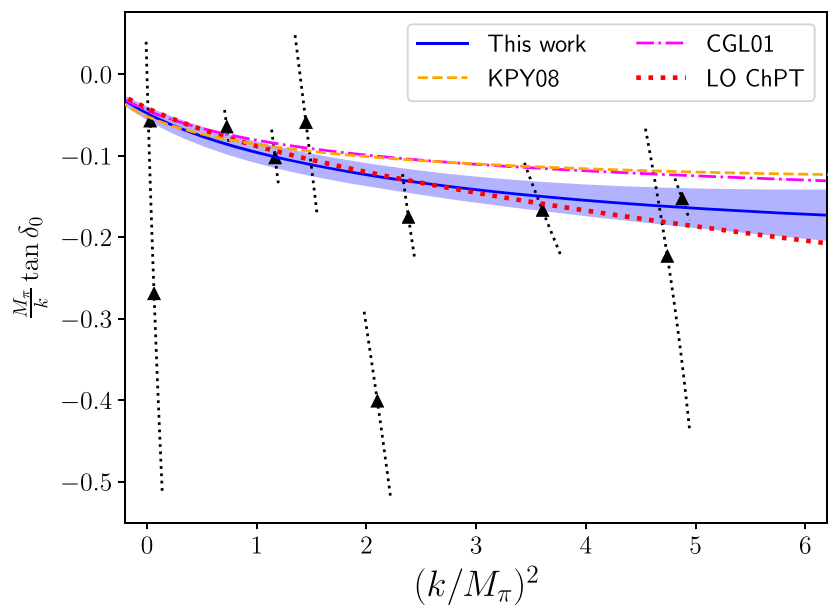

(a) $s$-wave

Fig. $2 s$ - and $d$-wave phase shift at the physical point (ensemble cA2.09.48) compared to the fits to experimental data (KPY08) in Ref. [92] and (CGL01) in [93]. For $s$-wave we use a model that incorporates

spondence of an energy level to a phase shift point [6,7]. For the analysis, we need an appropriate phase shift parametrization. We use a model that incorporates the expected Adler zero $[69,94]$ :

$\frac{k}{M_{\pi}} \cot \delta_{0}=\frac{\sqrt{s} M_{\pi}}{\left(s-2 z^{2}\right)}\left(B_{0}+B_{1} \frac{k^{2}}{M_{\pi}^{2}}+\cdots\right)$,

with $s$ the center-of-mass energy squared and $k^{2}=s / 4-M_{\pi}^{2}$. We will fix the position of the Adler zero to its leading order chiral perturbation theory (LO ChPT) value: $z^{2}=M_{\pi}^{2}$. Even though higher order corrections are to be expected, its value has been seen to be compatible with LO ChPT when left free $[92,95,96]$. Note that in Eq. 6 with fixed Adler zero, we have $M_{\pi} a_{0}=1 / B_{0}$.

We perform a correlated two-parameter fit to the energy levels. The results for the three ensembles are shown in Table 2. In all cases, the magnitude of the $B_{i}$ coefficients decreases with increasing order, indicating that the expansion converges quickly enough even at the heaviest pion mass. Still, for the heaviest ensemble (cA2.60.32), we also attempt a fit with a quadratic term in $k^{2}, B_{2}$ and observe a small, barely significant value for $B_{2}$ and no substantial change in $B_{0}$ and

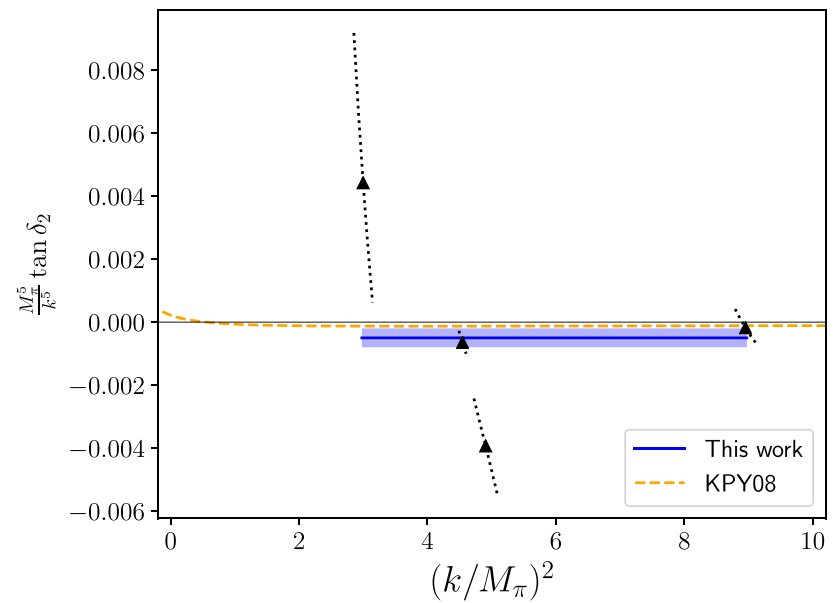

(b) $d$-wave

the Adler-zero, whereas for $d$-wave we fit to a constant in the region for which we have data

$B_{1}$. Based on ChPT, better convergence is expected for lighter pions.

The $s$-wave phase shift is visualised for the physical point ensemble in the left panel of Fig. 2. In this plot we also compare to other results in the literature. For the other two ensembles the corresponding plots can be found in the left panels of Figs. 9 and 10, respectively, in the appendix.

One interesting point to discuss is the suitability of the $\delta_{0}$ parametrization. It has been customary to use a standard effective range expansion parametrization (ERE) for isospin$2 \pi \pi$ scattering:

$$
\begin{aligned}
\frac{k}{M_{\pi}} \cot \delta_{0}= & \frac{1}{M_{\pi} a_{0}}+\frac{1}{2} M_{\pi} r\left(\frac{k}{M_{\pi}}\right)^{2} \\
& +M_{\pi}^{3} P\left(\frac{k}{M_{\pi}}\right)^{4} .
\end{aligned}
$$

However, the presence of the Adler zero limits the radius of convergence to $k^{2} \sim 0.5 M_{\pi}^{2}$. For this reason, explicitly incorporating the Adler zero must improve the radius of convergence, and has been shown to provide a better description of the data [69]. Here, we compare again the two fit models. The ERE results are shown in Table 3. As can be seen, the 
values of $\chi^{2}$ in the case of the ERE fits are always larger than their Adler-zero counterparts given in Table 2. This further supports the usage of the Adler-zero parametrization for $I=2 \pi \pi$ scattering.

Similarly, the $d$-wave phase shift can be obtained from most of the nontrivial irreps when neglecting $\ell>2$ waves $[15,16]$. Since we have few data points, we attempt the following fit (see Table 4):

$$
\frac{k^{5}}{M_{\pi}^{5}} \cot \delta_{2}=\frac{1}{M_{\pi}^{5} a_{2}} .
$$

The best fit curve for the physical point ensemble is show in the right panel of Fig. 2 and compared to Ref. [92]. Again, for the other two ensembles the corresponding plots can be found in the appendix in the right panels of Figs. 9 and 10, respectively.

In the three pion case we need to parametrize $\mathcal{K}_{3 \text {,df. For }}$ this, we expand $\mathcal{K}_{3, \mathrm{df}}$ about threshold up to linear terms of relativistic invariants [49]:

$\mathcal{K}_{3, \mathrm{df}}=\mathcal{K}_{\mathrm{df}, 3}^{\mathrm{iso}, 0}+\mathcal{K}_{\mathrm{df}, 3}^{\mathrm{iso}, 1} \Delta, \quad \Delta=\frac{\left(E^{*}\right)^{2}-9 M_{\pi}^{2}}{9 M_{\pi}^{2}}$,

where $\mathcal{K}_{\mathrm{df}, 3}^{\mathrm{iso}, 0}$ and $\mathcal{K}_{\mathrm{df}, 3}^{\mathrm{iso}, 1}$ are the numerical constants to be determined. This parametrization has no momentum dependence, and thus receives the name "isotropic". It is the threeparticle equivalent of keeping only $s$-wave interactions. At the next order in the expansion, $O\left(\Delta^{2}\right)$, three new parameters arise, for which also the $d$-wave must be included [49]. This is beyond the scope of the present analysis (Table 5).

Following the strategy outlined in Ref. [69], we perform a simultaneous $s$-wave only fit to two- $\pi^{+} A_{1}$ levels, and all three- $\pi^{+}$levels. For this, we use the $\delta_{0}$ model in Eq. 6 and the $\mathcal{K}_{3 \text {,df }}$ parametrization in Eq. 9 - four parameters in total, see Table 6 . As can be seen the best fit values for $B_{0}$ and $B_{1}$ agree well between the two-particle and the global fit, with even smaller errors in the case of the latter. For convenience, we provide the full covariance matrices of the fits in Table 6 in the appendix, see Eqs. (B2) to (B4).

We have also performed fits including only the constant term $\mathcal{K}_{\mathrm{df}, 3}^{\mathrm{iss}, 0}$, the results of which can be found in the appendix. We observe that for the ensembles with larger than physical pion mass value the inclusion of the linear term seems necessary.

In Fig. 11 in the appendix we provide as an example for the physical point ensemble the measured energy spectrum in the two- and three particle sectors separately. In that figure we also compare to the noninteracting energy levels. Moreover, we give the energy levels predicted by our fits, see Tables 2 , 4,6

\section{Discussion}

Starting with $\delta_{0}$, we show in Fig. 2a all phase shift data points, and include the best fit curve from the two- and three$\pi^{+}$global fit. As can be seen, the difference to LO ChPT is small, and due to $B_{1} \neq 0$. In addition, our results agree within $<2 \sigma$ with Refs. [92,93]. We obtain $M_{\pi} a_{0}=-0.0481(86)$ (see Table 6 and recall $1 / B_{0}=M_{\pi} a_{0}$ ), which also agrees well with all phenomenological determinations $[92,93,95-$ 98], and other lattice results obtained indirectly by extrapolating to the physical point using ChPT [3,17,34,99-105], see Fig. 1.

In Fig. 1 we also compare to results from $N_{f}=2+1+1$ calculations from Ref. [3] and with LO ChPT. Within the uncertainties we do not observe a significant difference between $N_{f}=2$ and $N_{f}=2+1+1$ results. Moreover, as was found in all previous investigations of two pions at maximal isospin, LO ChPT describes the mass dependence extraordinarily well. At the physical point, LO ChPT predicts $M_{\pi} a_{0} \simeq-0.04438$, which agrees within error bars with the value we report here, see above. Unfortunately, our determination here suffers from relatively large statistical uncertainties and, thus, cannot compete with determinations based on chiral extrapolations. A summary of various determinations from the literature is compiled in Table 5.

Regarding the $d$-wave phase shift, we have mild statistical evidence that it is repulsive at the physical point in the considered energy region. We observe agreement within $\gtrsim 1 \sigma$ with Ref. [92], as shown in Fig. 2b. An interesting feature of the phenomenological fits to $\delta_{2}$ is that there is a sign change near threshold, which yields an attractive phase shift at threshold $[92,95,96,106]$. We cannot confirm or deny such behaviour, as the explored energy region is too far above threshold. For larger pion mass values, we obtain a similar behaviour. The $d$-wave phase shift is more repulsive for the two larger pion mass values - see Table 4 and the appendix.

We show our results in the three-particle sector in Fig. 3. As can be seen in Fig. 3a, there is significant evidence that $\mathcal{K}_{3, \mathrm{df}}$ at threshold $\left(\mathcal{K}_{\mathrm{df}, 3}^{\mathrm{iso}, 3}\right)$ is positive (attractive). Even though we find reasonable agreement with the LO ChPT [69] prediction, the data suggests that NLO effects can be significant, and it may be worth to extend the ChPT result to one loop in future work. For $\mathcal{K}_{\mathrm{df}, 3}^{\mathrm{iso}, 1}$, the situation is somewhat different. All evidence points to a negative value, very far from the ChPT results. While one could conclude that a NLO ChPT description is required, there is a subtlety in the LO ChPT prediction: it assumes that the connection between $\mathcal{K}_{3 \text {,df }}$ and $\mathcal{M}_{3}$ - which involves integral equations - is trivial in LO ChPT [69]

$\mathcal{K}_{3, \mathrm{df}}=\mathcal{M}_{3, \mathrm{df}}\left[1+O\left(M_{\pi}^{2} / F_{\pi}^{2}\right)\right]$, 
Table 3 Two-particle fits to the standard effective range expansion (ERE) model in Eq. 7

\begin{tabular}{lllll}
\hline Ensemble & $M_{\pi} a_{0}$ & $M_{\pi} r$ & $M_{\pi}^{3} P$ & $\chi^{2} / \mathrm{dof}$ \\
\hline cA2.60.32 & $-0.2198(55)$ & $1.1(2)$ & - & $28.12 /(16-2)$ \\
cA2.60.32 & $-0.2177(56)$ & $2.1(5)$ & $-0.16(8)$ & $24.26 /(16-3)$ \\
cA2.30.48 & $-0.186(15)$ & $1.5(4)$ & - & $31.98 /(16-2)$ \\
cA2.09.48 & $-0.064(11)$ & $3.9(1.1)$ & - & $14.00 /(10-2)$ \\
\hline
\end{tabular}

Table $4 d$-wave two-pion fits to Eq. 8. Here we use only non- $A_{1}$ two-pion levels. The last column shows the energy range for which data is used

\begin{tabular}{lllr}
\hline & $M_{\pi}^{5} a_{2}$ & $\chi^{2} /$ dof & CM energy range \\
\hline cA2.60.32 & $-0.0037(08)$ & $15.03 /(12-1)$ & {$\left[3.2 M_{\pi}, 4.4 M_{\pi}\right]$} \\
cA2.30.48 & $-0.0072(11)$ & $23.78 /(10-1)$ & {$\left[2.8 M_{\pi}, 4.2 M_{\pi}\right]$} \\
cA2.09.48 & $-0.0005(03)$ & $7.33 /(4-1)$ & {$\left[4.0 M_{\pi}, 6.3 M_{\pi}\right]$} \\
\hline
\end{tabular}

Table 5 Summary of some lattice and phenomenological determinations of the isospin- $2 \pi \pi$ scattering length at the physical point. Note that the lattice determination of ETM (2015) is the only one with chiral and continuum extrapolations. We list LO ChPT, ChPT and Roy equations [93] denoted as CGL01, CCL11 [97], CP-PACS [99], NPLQCD (2006) [100], NPLQCD (2008) [101], ETM (2013) [17], ETM (2015) [3], Yagi et al. [103], Fu [104] and PACS-CS [105], and GWU [34]

\begin{tabular}{lll}
\hline & $N_{f}$ & $M_{\pi} a_{0}$ \\
\hline LO ChPT & & -0.04438 \\
CGL01 (2001) & & $-0.0444(10)$ \\
CCL11 (2011) & & $-0.0445(14)$ \\
CP-PACS (2004) & 2 & $-0.0431(29)(-)$ \\
NPLQCD (2006) & $2+1$ & $-0.0426(6)(3)$ \\
NPLQCD (2008) & $2+1$ & $-0.04330(42)$ comb \\
ETM (2010) & 2 & $-0.04385(28)(38)$ \\
ETM (2015) & $2+1+1$ & $-0.0442(2)\left({ }_{-0}^{+4}\right)$ \\
Yagi (2011) & 2 & $-0.04410(69)(18)$ \\
Fu (2013) & $2+1$ & $-0.04430(25)(40)$ \\
PACS-CS (2014) & $2+1$ & $-0.04263(22)(41)$ \\
GWU (2019) & 2 & $-0.0433(2)$ \\
This work & 2 & $-0.0481(86)(-)$ \\
\hline
\end{tabular}

where $\mathcal{M}_{3 \text {,df }}$ is the divergence-free three-to-three amplitude [43]. As argued in Ref. [69], this induces large errors in $\mathcal{K}_{\mathrm{df}, 3}^{\mathrm{iso}, 1}$ (up to $50 \%$ for $200 \mathrm{MeV}$ pions). The situation is expected to be more dramatic for heavier pions, like our two results at 242 and $340 \mathrm{MeV}$, for which the largest difference is seen. In order to address this rigorously, the integral equation must be systematically solved, which is beyond the scope of this work.

\section{Conclusion}

We have presented the first $N_{f}=2$ lattice calculation of twoand three- $\pi^{+}$scattering at the physical point. In the two pion channel we observe very good agreement with other lattice calculations and ChPT or ChPT combined with Roy-Steiner equations for the $s$-wave phase shift. In particular, for the whole range of pion mass values we have available here we do not observe a significant deviation from LO ChPT or a significant difference to $N_{f}=2+1+1$ lattice results. For the $d$-wave our uncertainties are relatively large. However, thanks to the physical point ensemble we can directly compare to phenomenology and observe reasonable agreement. For the $d$-wave phase shift smaller scattering momenta would

Table 6 Two- and three-pion fits using the Adler-zero form $\left(z^{2}=M_{\pi}^{2}\right.$, fixed). Since we only include $s$-wave interactions, we use two-pion levels in the $A_{1}$ irrep, and all irreps for three-pions. Recall that $1 / B_{0}=M_{\pi} a_{0}$

\begin{tabular}{llllllr}
\hline & $M_{\pi} a_{0}$ & $B_{1}$ & $B_{2}$ & $M_{\pi}^{2} \mathcal{K}_{\mathrm{df}, 3}^{\text {iso, }, 3}$ & $M_{\pi}^{2} \mathcal{K}_{\mathrm{df}, 3}^{\text {iso, }}$ & $\chi^{2} / \mathrm{dof}$ \\
\hline cA2.60.32 & $-0.2061(49)$ & $-1.9(2)$ & - & $4500(1500)$ & $-6200(1800)$ & $58.89 /(43-4)$ \\
cA2.60.32 & $-0.2070(52)$ & $-2.2(5)$ & $0.1(2)$ & $4300(1500)$ & $-6000(1800)$ & $58.50 /(43-5)$ \\
cA2.30.48 & $-0.156(15)$ & $-1.9(4)$ & - & $1800(3800)$ & $-4300(3800)$ & $46.18 /(33-4)$ \\
cA2.09.48 & $-0.0481(86)$ & $-1.3(1.1)$ & - & $0(800)$ & $-200(500)$ & $19.06 /(19-4)$ \\
\hline
\end{tabular}




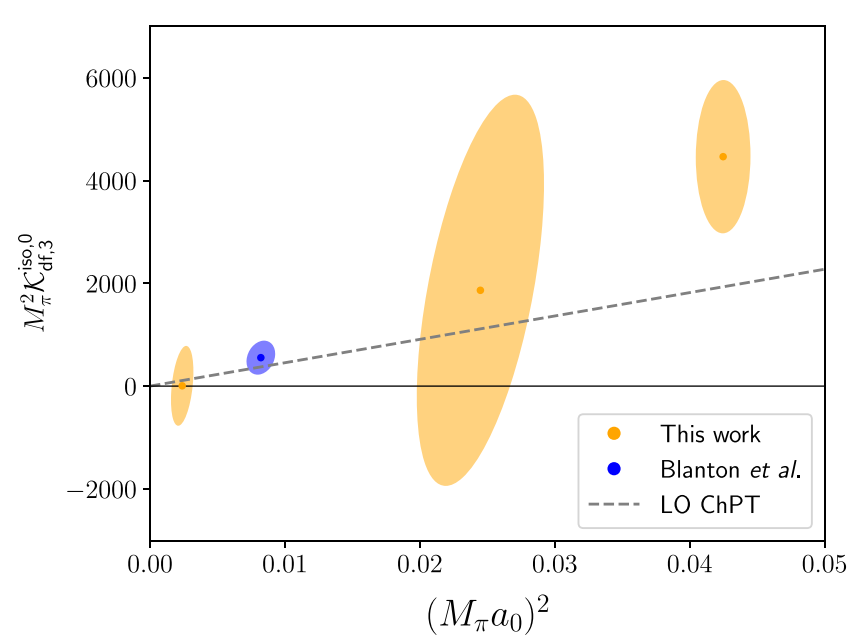

(a) $\mathcal{K}_{\mathrm{df}, 3}^{\mathrm{iso}, 0}$

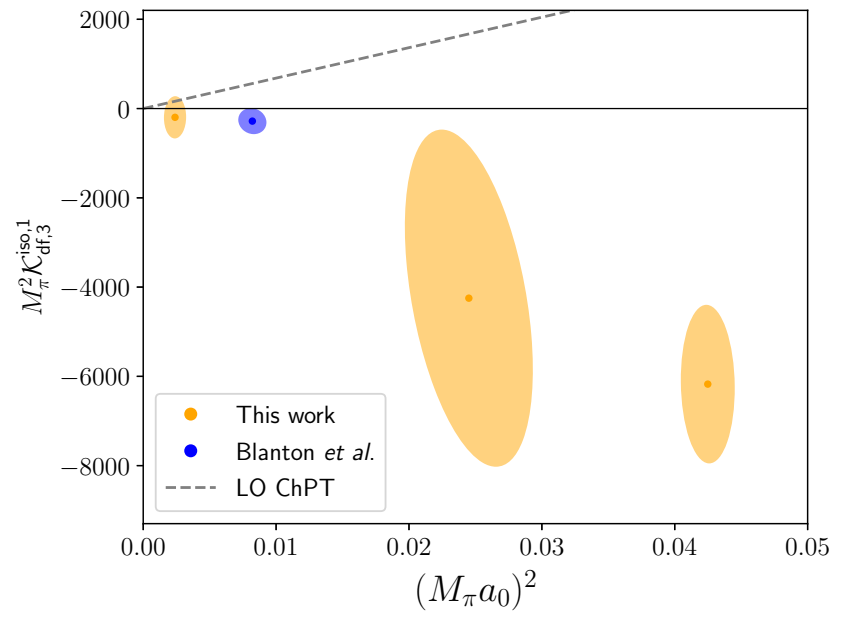

(b) $\mathcal{K}_{\mathrm{df}, 3}^{\text {iso, } 1}$

Fig. 3 Constant(left) and linear(right) terms of $\mathcal{K}_{3 \text {,df }}$ as a function of the $s$-wave scattering length. We also include the results of Ref. [69]

be desirable in order to be able to shed light on a possible sign change at small $k^{2}$-values.

For the three pion case, we observe reasonable agreement with other lattice calculations, phenomenology, and ChPT. By including two ensembles at heavier pion masses, we have gained insight on the chiral dependence of three- $\pi^{+}$scattering quantities for the first time. We use an isotropic parametrisation of $\mathcal{K}_{3 \text {,df }}$, the real, singularity free, quasilocal, intermediate three particle scattering quantity. Here we find good agreement to LO ChPT for the constant term in $\mathcal{K}_{3 \text {,df }}$ in an expansion about threshold, but an opposite sign compared to LO ChPT for the next-to-leading term. We have discussed possible explanations for this. On the other hand, qualitative agreement is found for both terms with the other available lattice calculation of these quantities.

This letter represents a step towards exploring and understanding the hadronic spectrum of QCD, and shows that three-particle quantities can be extracted with current techniques. In the very near future we expect more lattice calculations of three-body observables with increasing accuracy and describing systems with growing complexity - e.g. threeparticle resonances such as the $\omega$.

Acknowledgements We thank all members of ETMC for the most enjoyable collaboration. We also thank P. Hernández, A. Rusetsky, and S. Sharpe for comments on the manuscript. The authors gratefully acknowledge the Gauss Centre for Supercomputing e.V. (http://www. gauss-centre.eu) for funding this project by providing computing time on the GCS Supercomputer JUQUEEN [107] and the John von Neumann Institute for Computing (NIC) for computing time provided on the supercomputers JURECA [108] and JUWELS [109] at Jülich Supercomputing Centre (JSC). Parts of the the results were created within the EA program of JUWELS Booster and we are thankful for the help and support of the JUWELS Booster Project Team (JSC, Atos, ParTec, NVIDIA). This project was funded in part by the DFG as a project in the Sino-German CRC110. FRL acknowledges the support provided by the European projects H2020-MSCA-ITN-2015/674896-ELUSIVES, H2020-MSCA-RISE-2015/690575-InvisiblesPlus, the Spanish project FPA2017-85985-P, and the Generalitat Valenciana grant PROMETEO/2019/083. The work of FRL also received funding from the EU Horizon 2020 research and innovation program under the Marie Skłodowska-Curie Grant Agreement No. 713673 and "La Caixa" Foundation (ID 100010434, LCF/BQ/IN17/11620044). The open source software packages tmLQCD [110-112], Lemon [113], QUDA [114116], R [117], hadron [118] and paramvalf [119] have been used.

Data Availability Statement This manuscript has associated data in a data repository. [Authors' comment: The energy levels of this work can be found in a GitHub repository [91].]

Open Access This article is licensed under a Creative Commons Attribution 4.0 International License, which permits use, sharing, adaptation, distribution and reproduction in any medium or format, as long as you give appropriate credit to the original author(s) and the source, provide a link to the Creative Commons licence, and indicate if changes were made. The images or other third party material in this article are included in the article's Creative Commons licence, unless indicated otherwise in a credit line to the material. If material is not included in the article's Creative Commons licence and your intended use is not permitted by statutory regulation or exceeds the permitted use, you will need to obtain permission directly from the copyright holder. To view a copy of this licence, visit http://creativecomm ons.org/licenses/by/4.0/.

Funded by $\mathrm{SCOAP}^{3}$.

\section{Appendix A: Extraction of the energy levels}

In this section, we provide more details regarding the extraction of energy levels from the correlation functions of one, two and three charged pions. All the required quark contraction diagrams are shown in Fig. 4. For the observables in question we have determined the integrated autocorrelation times using the method put forward in [120] and found that 
we can treat our measurements as decorrelated. The statistical analysis is performed via bootstrap.

\section{A.1 Thermal pollutions}

Given the individual pion momenta $p_{i}, i=1,2,3$, we adopt the following convention to express the total momentum $\mathbf{P}=$ $\sum_{i} \mathbf{p}_{\mathbf{i}}$ and the relative momenta $\mathbf{q}_{j}, j=1,2$

$\mathbf{p}_{1}=\mathbf{P}-\mathbf{q}_{1}-\mathbf{q}_{2}, \quad \mathbf{p}_{2}=\mathbf{q}_{1}, \quad \mathbf{p}_{3}=\mathbf{q}_{2}$.

The spectral composition of a three-pion correlation function (with periodic boundary conditions) reads

$$
\begin{aligned}
\sum_{m} \sum_{n}\left\langle n\left|O_{\Gamma}\left(\mathbf{P}, \mathbf{q}_{1}, \mathbf{q}_{2}\right)\right| m\right\rangle\left\langle m\left|O_{\Gamma}^{\dagger}\left(\mathbf{P}, \mathbf{q}_{3}, \mathbf{q}_{4}\right)\right| n\right\rangle \\
\mathrm{e}^{-E_{n} \cdot(T-t)} \mathrm{e}^{-E_{m} t} .
\end{aligned}
$$

The double sum is over all states $m, n$ with the correct quantum numbers. The desired signal arises when $m$ is the vacuum, and $n$ the three-pion state, or vice versa. Usually one would expect that all other contributing to the spectral decomposition are exponentially suppressed compared to this ground state. Here this is not the case, because there are nonzero contributions to the spectral decomposition for finite $T$ for instance when $m$ is an intermediate two-pion state and $n$ is a one-pion state. Such so-called thermal pollution states have a time dependence proportional to $\exp (-\Delta E t)$, with $\Delta E=E_{2 \pi}-E_{\pi}$, which can dominate the correlation function for large enough $t$ when $\Delta E<E_{3 \pi}$. There is an additional backward propagating part as well which goes as $\exp (-\Delta E \cdot(T-t))$. Together they either form a cosh (sum) or a sinh (difference). For three pions we only have time-even operators and therefore everything will have a cosh-shape. The amplitude of the cosh will be proportional to $\exp \left(-\left(E_{2 \pi}+E_{\pi}\right) T\right)$, which vanishes for $T \rightarrow \infty$.

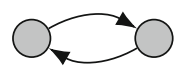

(a) $\mathrm{C} 2 \mathrm{c}$

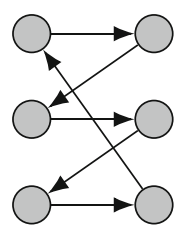

(d) $\mathrm{C} 6 \mathrm{cC}$

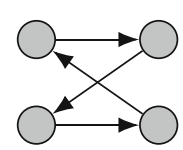

(b) $\mathrm{C} 4 \mathrm{cC}$

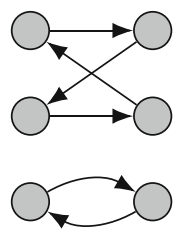

(e) $\mathrm{C} 6 \mathrm{cCD}$

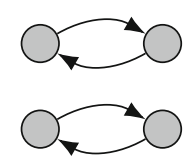

(c) $\mathrm{C} 4 \mathrm{cD}$

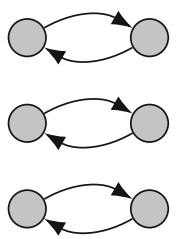

(f) $\mathrm{C} 6 \mathrm{cD}$
Fig. 4 Quark contraction diagrams for the one-, two- and three- $\pi^{+}$ correlation functions needed in this work
The size of the pollution will depend on the individual momenta of the involved pions through the energy $E_{2 \pi}$ and $E_{\pi}$. The most significant pollution will be the one leading to the smallest $\Delta E$, which usually corresponds to the smallest involved momenta.

The thermal pollutions depend also on the frame and irrep. Let us illustrate this for a specific example: assume that $n$ is a one-pion state $\left|\mathbf{p}_{1}\right\rangle$ and $m$ a two-pion state $\left|\mathbf{p}_{2}, \mathbf{p}_{3}\right\rangle$ with free energies given by the dispersion relation. In this specific case only summands where $\left\langle\mathbf{p}_{1}\left|O_{\Gamma}\right| \mathbf{p}_{2}, \mathbf{p}_{3}\right\rangle \neq 0$ contribute, i.e. the three-pion operator $O_{\Gamma}$ must couple to the momenta $\mathbf{p}_{1}, \mathbf{p}_{2}$ and $\mathbf{p}_{3}$.

The individual particle momenta that couple to a multiparticle operator can be inferred from group theory. Consider the frame $\mathbf{P}^{2}=0$, then the three-pion operator will be in some irrep $\Gamma^{-}$, and the single pion always in the $A_{1}^{-}$. Therefore, the two-pion system needs to be in the opposite parity irrep $\Gamma^{+}$such that $A_{1}^{-} \otimes \Gamma^{+}=\Gamma^{-}$. Note that only the irreps for $\mathbf{P}^{2}=0$ have a parity index, that is, in moving frames parity is not a good quantum number. In this situation, the momenta of the two-pion system can only take the values that actually couple to the irrep of the three-particle operator.

The allowed contributions are generated from all permutations of the three-pion individual momenta. Using the measured pion rest mass $M_{\pi}$ and the free particle dispersion relation (assuming weak interactions between the two pions) we can thus estimate the relevant energies $E_{\pi}\left(\mathbf{p}_{1}\right)$ and $E_{2 \pi}\left(\mathbf{p}_{2}, \mathbf{p}_{3}\right)$. Using these together with the $T$-values we can now estimate for every ensemble, irrep and total momentum which thermal contribution is - up to unknown matrix elements - largest. Since we are able to remove only a single thermal state, this is the only way to single out the relevant parameters for the possible subtraction of these polluting states. Figure 5 shows the contributing thermal states for two example cases, left the $A_{1}^{-}$irrep in the $\mathbf{P}^{2}=0$ frame, right the $B_{1}$ irrep in the $\mathbf{P}^{2}=2$ frame. The different correlators shown correspond to different combinations of single and two pion momenta. For these cases the largest contribution is coming from $\left(\mathbf{p}_{1}^{2}=0, \mathbf{p}_{2}^{2}=0, \mathbf{p}_{3}^{2}=0\right)$ and $\left(\mathbf{p}_{1}^{2}=1, \mathbf{p}_{2}^{2}=2, \mathbf{p}_{3}^{2}=1\right)$, respectively. The other possible contributions are suppressed by two orders of magnitude or even exponentially.

To be precise, in order to find the dominating contribution for each irrep, ensemble and frame, we take the largest thermal contribution at $t=10$, from which we can estimate $\Delta E$. To illustrate this procedure further, we will look at irrep $\Gamma=B_{1}$ with $\mathbf{P}^{2}=2$. The three-particle momenta that couple to the operator below our threshold are listed in Table 7. As the three particles are indistinguishable, we can partition them at will into a one-pion and two-pion state. The twoparticle momenta must again be a valid two-particle system, otherwise they cannot be an intermediate thermal state. Table 8 lists the two-particle contributions in the $B_{1}$ irrep. 


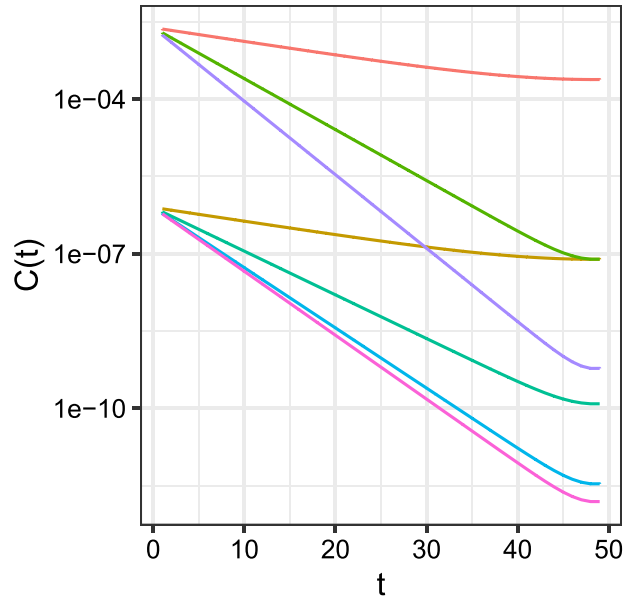

(a) $\mathbf{P}^{2}=0$ in $A_{1}^{-}$

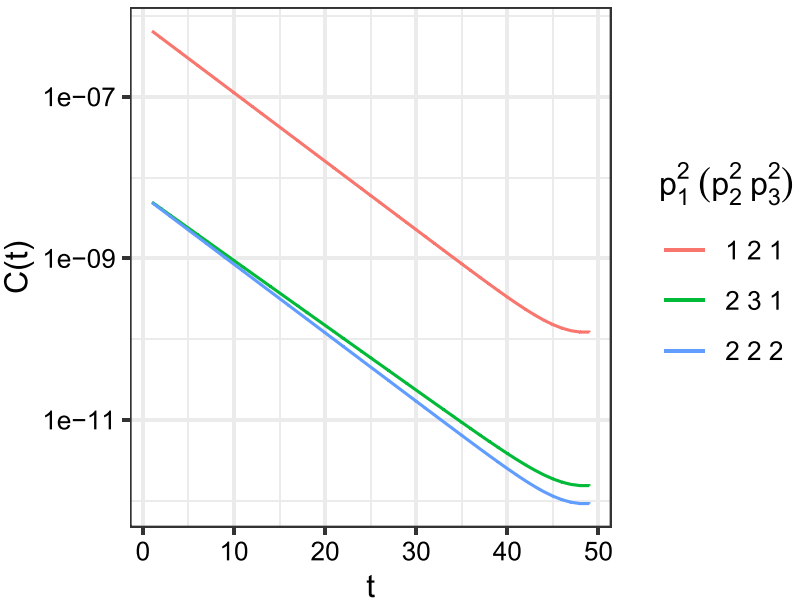

(b) $\mathbf{P}^{2}=2$ in $B_{1}$

Fig. 5 Possible thermal contributions to the three-pion correlator matrix in the cA2.09.48 ensemble. Each line corresponds to a particular combination of the individual particle momentum magnitudes of the one-pion $\left(\mathbf{p}_{1}\right)$ and the two-pion system $\left(\mathbf{p}_{2}\right.$ and $\left.\mathbf{p}_{3}\right)$

Table 7 Possible three-pion individual momenta in the $\Gamma=B_{1}$ irrep with total momentum $\mathbf{P}^{2}=2$

\begin{tabular}{lllll}
\hline $\mathbf{P}^{2}$ & Irrep & $\mathbf{p}_{1}$ & $\mathbf{p}_{2}$ & $\mathbf{p}_{3}$ \\
\hline 2 & $B_{1}$ & $(0,1,-1)$ & $(0,0,1)$ & $(1,0,0)$ \\
2 & $B_{1}$ & $(1,1,0)$ & $(1,0,0)$ & $(-1,0,0)$ \\
2 & $B_{1}$ & $(-1,1,0)$ & $(1,0,0)$ & $(1,0,0)$ \\
\hline
\end{tabular}

Table 8 Possible two-pion individual momenta in the $B_{1}$ irrep for different values of $\mathbf{P}^{2}$ of the two-pion subsystem

\begin{tabular}{llll}
\hline $\mathbf{P}^{2}$ & Irrep & $\mathbf{p}_{2}$ & $\mathbf{p}_{3}$ \\
\hline 1 & $B_{1}$ & $(-1,0,1)$ & $(1,0,0)$ \\
2 & $B_{1}$ & $(1,1,-1)$ & $(0,0,1)$ \\
4 & $B_{1}$ & $(0,-1,1)$ & $(0,1,1)$ \\
\hline
\end{tabular}

Thus, again for the example of the $B_{1}$ irrep, we have to go through the following possibilities:

- We take $(0,1,-1)$ for the one pion and $(0,0,1)$ and $(1,0,0)$ for the other two. The two-pion system has $\mathbf{P}^{2}=2$, but the lowest contribution in that irrep has larger momenta. So this does not contribute.

- The single pion has $\mathbf{p}_{1}=(1,1,0)$ and the two-pion system gets $\mathbf{p}_{2}=(1,0,0)$ and $\mathbf{p}_{3}=(-1,0,0)$. The two-pion system therefore has total momentum $\mathbf{P}^{2}=0$, but there is no contribution to $B_{1}$ in that moving frame. Therefore this example does not contribute to the thermal states.

- A contribution is obtained using $(1,0,0)$ for the one pion momentum, and $(-1,1,0)$ and $(1,0,0)$ for the two-pion system. In the latter, we have $\mathbf{P}^{2}=1$, which corresponds to the first entry in Table 8 (albeit after an inconsequential global rotation). This contributes as a thermal state, incidentally it is the largest one as shown in Fig. 5 b.

Of course, there are many more possibilities to check for. Using this method we determine the leading thermal state for every correlator matrix and can use this as input for thermal state treatments, detailed below.

\section{A.2 General technicalities}

Multi-particle correlators in general are contaminated with excited states at early times, and with thermal pollution at late time slices. Fitting too early will overestimate the energy, while fitting too late may underestimate it. In order to obtain a robust energy estimate, we use combinations of different methods to attenuate these issues.

The order of application of these methods is illustrated with a flow chart in Fig. 6. The detour arrows indicate optional parts of the chain. We will explain the different methods in order. First the correlator matrices can optionally be treated with weight-shift-reweight [76] to suppress thermal states at the cost of larger statistical uncertainty. Then we independently use the original and treated correlator matrix and apply the GEVM, which yields the principal correlators. These principal correlators can be used to build ratios [17,31] or left as-is. All variants can optionally be fed into the Prony generalized Eigenvalue method (PGEVM) [90] with $t_{0}=2$ fixed to suppress excited states (The PGEVM with $\delta_{0}$ fixed, see Ref. [90] for details, turned out to not be reliable).

The resulting treated correlators are evaluated by looking at the so called effective mass. The simplest definition of it 


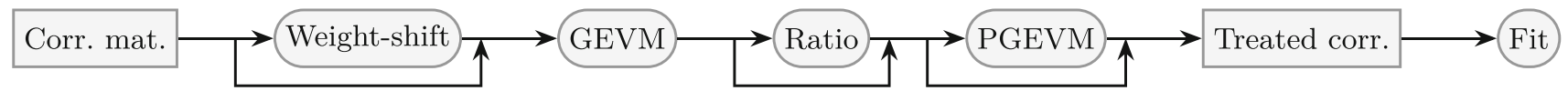

Fig. 6 Treatment of correlator matrices before fitting

is the "log effective mass"

$m_{\mathrm{eff}}(t)=-\log \frac{C(t)}{C(t+1)}$,

which assumes a signal proportional to $\exp (-E t)$ only. There are generalizations that take back-propagation, shifting or weighting into account. Depending on the treatment of the correlator we choose the appropriate effective mass.

We do not use all of the possible treatments in our analysis, but only the following five: no treatment (i.e. all optional parts are left out), only PGVM, only ratio, only weight and shift and finally the combination of weight and shift with PGEVM. In more detail this means:

No treatment When no thermal states contribute (like in $E$ irreps in the two pion channel), a simple cosh-like model is fitted:

$$
C(t)=A_{0}\left[\exp \left(-E_{0} t\right)+\exp \left(-E_{0} \cdot(T-t)\right)\right] .
$$

If thermal states are present in the given irrep, a two-state model

$$
\begin{aligned}
C(t)= & A_{0}\left[\exp \left(-E_{0} t\right)+\exp \left(-E_{0} \cdot(T-t)\right)\right] \\
& +A_{1}\left[\exp \left(-E_{1} t\right)+\exp \left(-E_{1} \cdot(T-t)\right)\right],
\end{aligned}
$$

with constrained second energy $E_{1}$ will be fitted to the data (for how $E_{1}$ and its error is determined, see Appendix A1) The constraint is implemented by augmenting the $\chi^{2}$ function to be minimized by a term

$\chi_{\mathrm{add}}^{2}=\left(-\frac{E_{1}-\bar{E}_{1}}{\delta E_{1}}\right)^{2}$,

where $E_{1}$ is the fit parameter, $\bar{E}_{1}$ is the determined central value for the thermal energy and $\delta E_{1}$ the statistical uncertainty on $E_{1}$.

PGEVM This method works well when there are no significant thermal state contributions. We fit a simple exponential model at early times.

Ratio We take the ratio of the principal correlator obtained from the GEVP (no weight-and-shift applied) and form ratios with the one-pion correlation function:

$R_{2}(t)=\frac{C_{2 \pi}(t)-C_{2 \pi}(t+1)}{C_{\pi}(t)^{2}-C_{\pi}(t+1)^{2}}$,

$$
R_{3}(t)=\frac{C_{3 \pi}(t) / C_{\pi}(t)-C_{3 \pi}(t+1) / C_{\pi}(t+1)}{C_{\pi}(t)^{2}-C_{\pi}(t+1)^{2}} .
$$

The ratio $R_{3}$ is chosen as a double ratio such that in the numerator, thermal state contributions $\propto \exp (-\Delta E t)$ are removed, since $\Delta E \approx E_{\pi}$. The resulting sinhlike correlator needs to be divided by another sinh-like expression, that's why we take the difference also in the denominator. Among different ratio expressions we have tested, this one works best in the sense that the plateau is longest. An exponential model is fitted to the ratios where the signal behaves like $R_{2}(t) \sim \exp \left(-\left(E_{2 \pi}-2 E_{\pi}\right) t\right)$ and $R_{3}(t) \sim \exp \left(-\left(E_{3 \pi}-3 E_{\pi}\right) t\right)$. Note that for the ratios we do not include backwards propagating parts and thus do not extend fit ranges too far towards $T / 2$.

Weight-shift The correlator matrix has the leading thermal state removed [76] and, therefore, the principal correlators can be fitted with a cosh-like model which incorporates the weight-shift-reweight procedure.

Weight-shift and PGEVM In general the additional suppression of excited states by the application of the PGEVM works well after weight-shift has been applied beforehand. The resulting correlator is fitted with an exponential model. Fit ranges can be chosen early enough such that the neglect of backwards propagating parts is not significant.

Figure 7 shows a comparison between no treatment, weight-shift and the ratio $R_{3}$ for a case with heavy thermal pollution. One can see how the effective mass of the plain correlator does not show any plateau due to the high degree of thermal pollution. The effective mass of the weighted correlator, however, exhibits a plateau between $t_{1}=12$ and $t_{2}=14$, but still shows a drop beyond. However, this three time slice plateau can only be identified when compared to the effective mass given by the ratio $R_{3}$. This likely stems from the second leading thermal state as visible in Fig. 5a. The ratio however has a long plateau that is compatible with the weight-shift method a posteriori. In general we see that with the ratio method it is possible to fit energy levels with strong thermal pollution when other methods fail to produce a plateau. The statistical uncertainty from the energy determination with the ratio is also lower than with other methods in most cases.

In some cases the thermal states are so pronounced that no plateau can be identified, even after applying the PGEVM. In these cases the method is not used for that particular level. 


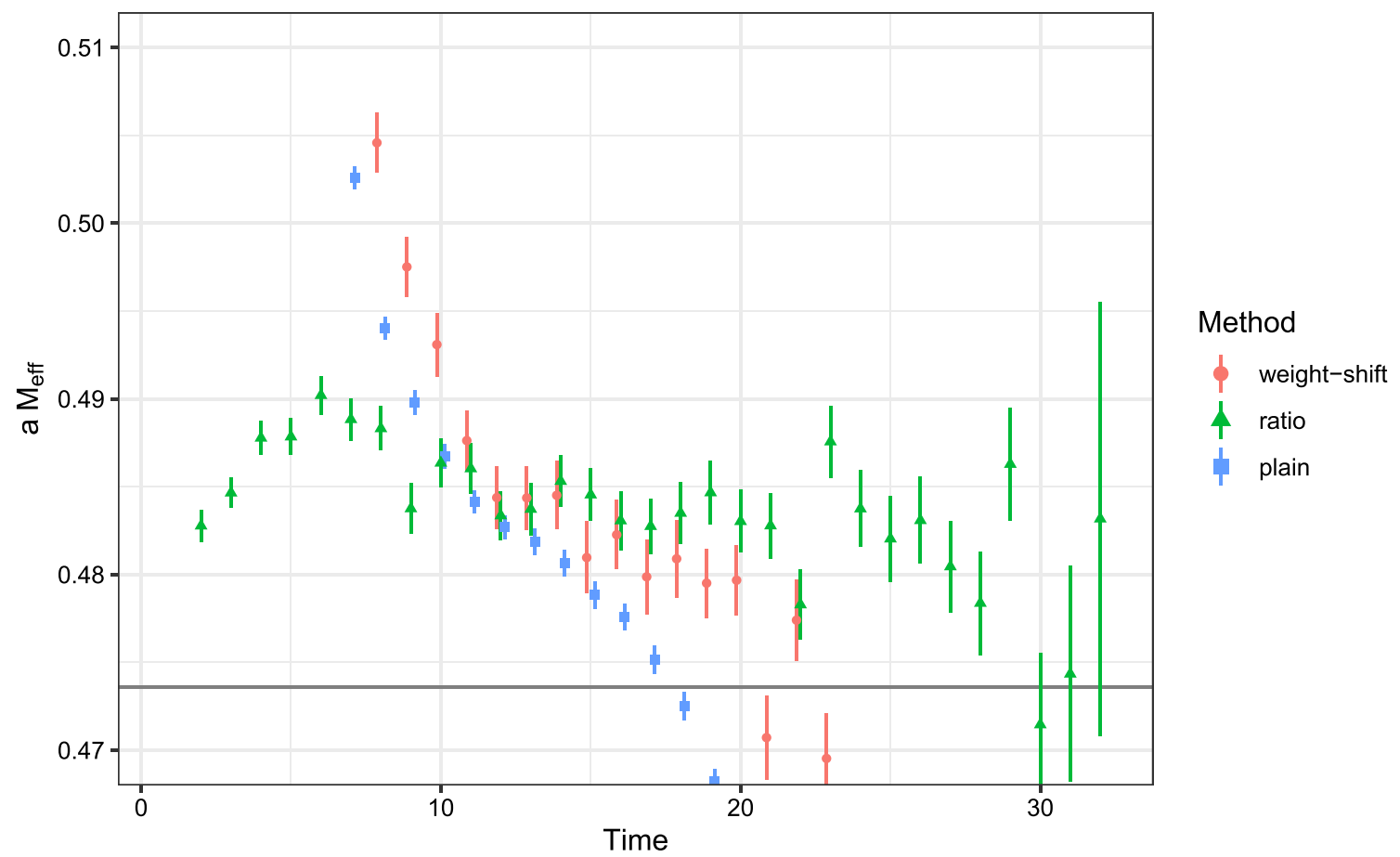

Fig. 7 Effective mass for the three-pion ground state $\left(A_{1}^{-}, \mathbf{P}^{2}=0\right)$ on the cA2.60.32 ensemble. Shown are in blue the plain correlator without shift-reweight and in green the ratio $R_{3}$ shifted upwards by $3 M_{\pi}$. The any thermal state treatment, in red the correlator treated with weightsolid line marks the noninteracting energy

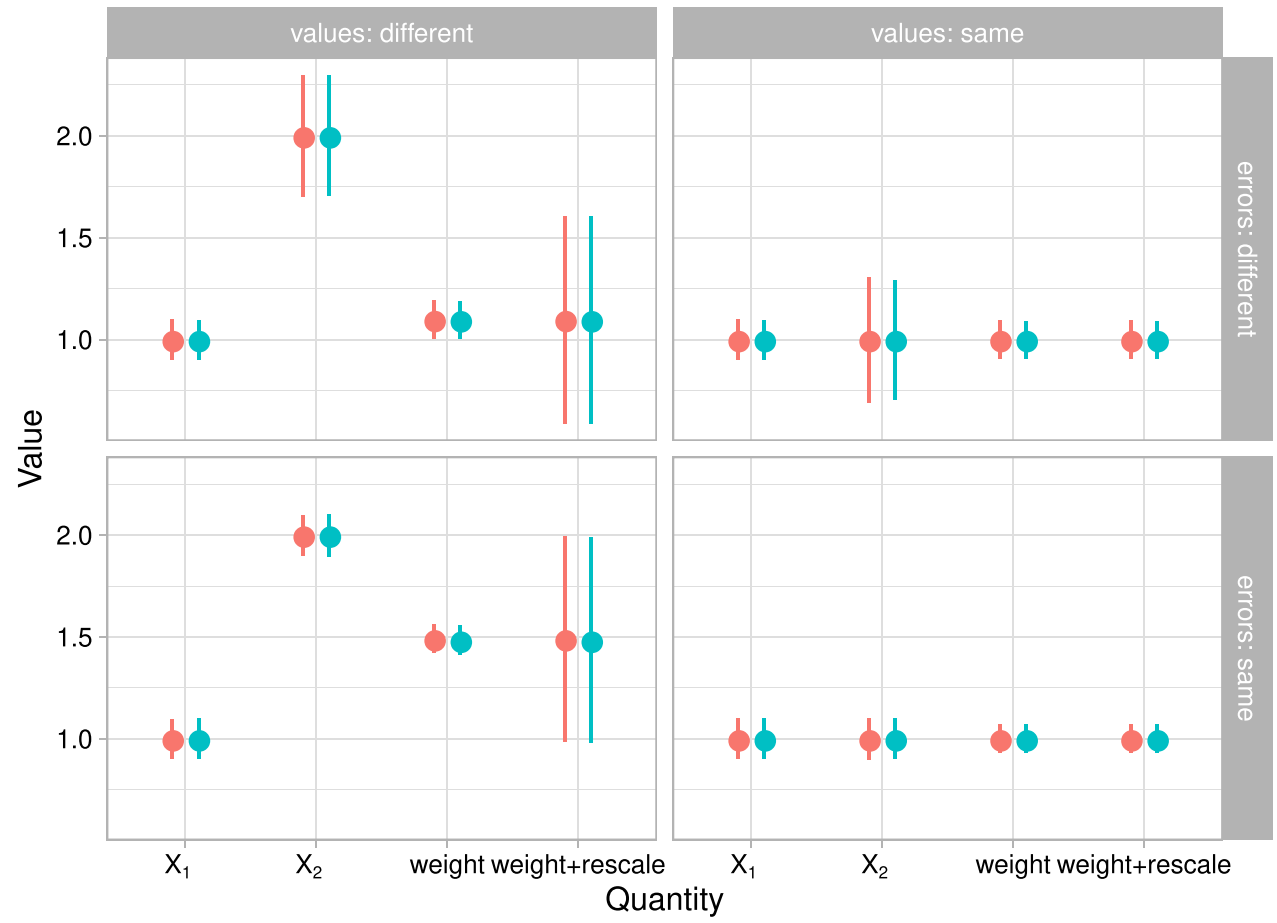

Resampling

bootstrap

jackknife different and same central values, rows show different and same statistical errors in the two measurements 
These cases work much better with either the multi-state model, weight-shift-reweight or the combination of weightshift-reweight and the PGEVM. The ratio method seems to be the most robust one, it shows plateaus even when other methods fail to produce one. Also, the statistical uncertainty seems to be lower compared to the other methods in general.

For every principal correlator we attempt to extract the energy with all the five methods detailed above. If a plateau can be identified, we use the extracted energy level. All such determinations per principal correlator are combined with a correlated weighted average. In order to incorporate the systematic spread between the central values, we also compute a systematic error scaling factor as introduced in Ref. [32]: for energy level $E$ we compute the scaling factor $w$ Eq. (5), as mentioned in the main text.

To illustrate this method to incorporate the systematic error into the resampling distribution, we use two artificially generated data points with central values $X_{1}$ and $X_{2}$ and corresponding standard errors generated in four ways, where either the central values and/or errors are chosen to be the same or different. All combinations thus give four cases, which are shown in the quadrants of Fig. 8 (upper left: different mean, different errors; upper right: same mean, different errors; lower left: different mean, same errors; lower right: all the same). The central values with standard errors for $X_{1}$ and $X_{2}$ are shown as the first two pairs of points in each quadrant. The third pair shows the weighted average of the two estimates and the fourth pair the result after the rescaling. One can nicely see how the weighted average gravitates toward the data point with the smaller uncertainty (hence higher weight) and how the rescaling incorporates the spread between the central values. The method works well for both bootstrap and jackknife resampling.

In order to choose appropriate fit ranges for the different methods, we proceed iteratively, selecting fit ranges by eye guided by the $p$-value of the fit. Energy levels are included in the further analysis only if a plateau of at least five time slices length could be identified for the $T=96$ lattices and of at least four time slices for the $T=64$ lattice. Some energy levels show significant tension between the different fitting methods after this first iteration. In these cases, we re-evaluate the plateaus to arrive at our final choices.

\section{Appendix B: Fitting the spectrum}

Here, we aim to extend the discussion of the fitting procedure of the spectrum to the quantization condition. The summary of the frames, irreps and energies used in this work is shown in Table 9.

Table 9 Summary of energy levels included in this work. The $E / M_{\pi}$ range indicates in which energy range the energy levels from the various principal correlators in that specific irrep where located

\begin{tabular}{lll}
\hline $\mathbf{P}^{2}$ & Irrep & $E / M_{\pi}$ range \\
\hline
\end{tabular}

(a) cA2.09.48, two pions

0

$0 \quad E^{+}$

$1 \quad A_{1}$

$2 \quad A_{1}$

$3 \quad A_{1}$

$3 \quad E$

$4 \quad A_{1}$

$4 \quad B_{1}$

(b) cA2.09.48, three pions

$\begin{array}{lll}0 & A_{1}^{-} & {[3.09,6.05]} \\ 0 & E^{-} & {[5.91,5.92]} \\ 1 & A_{2} & {[3.92,3.92]} \\ 2 & A_{2} & {[4.37,4.99]} \\ 3 & A_{2} & {[4.69,6.40]} \\ 3 & E & {[5.70,8.06]} \\ 4 & A_{2} & {[3.92,6.42]} \\ 4 & B_{2} & {[6.42,6.42]}\end{array}$

(c) cA2.30.48, two pions

0

$A_{1}^{+}$

[2.01, 3.99]

$[3.09,3.88]$

$[2.29,4.15]$

[3.28, 3.28]

$[4.09,4.09]$

[3.31, 4.02]

$[2.46,4.26]$

$[3.50,3.50]$

$[3.45,3.45]$

$[4.22,4.22]$

$[2.59,4.46]$

[2.84, 4.44]

[2.03, 3.13]

$[3.12,3.12]$

(d) cA2.30.48, three pions

0

0

1

1

1

2

2

2

2

3

3
$[3.05,4.26]$

[4.16, 4.16]

$[3.39,4.66]$

[4.43, 4.59]

[4.43, 4.43]

$[4.69,4.85]$

$[3.66,5.01]$

[4.81, 4.90]

$[4.59,4.90]$

[5.14, 5.14]

$[3.83,5.32]$ 
Table 9 continued

\begin{tabular}{|c|c|c|}
\hline$\overline{\mathbf{P}^{2}}$ & Irrep & $E / M_{\pi}$ range \\
\hline 3 & $E$ & {$[4.11,5.18]$} \\
\hline 4 & $A_{2}$ & {$[3.42,4.67]$} \\
\hline 4 & $B_{2}$ & {$[4.41,4.56]$} \\
\hline 4 & $E$ & {$[4.57,4.57]$} \\
\hline \multicolumn{3}{|c|}{ (e) cA2.60.32, two pions } \\
\hline 0 & $A_{1}^{+}$ & {$[2.02,4.20]$} \\
\hline 0 & $E^{+}$ & {$[3.21,4.08]$} \\
\hline 1 & $A_{1}$ & {$[2.32,4.38]$} \\
\hline 1 & $B_{1}$ & {$[3.43,3.43]$} \\
\hline 1 & $B_{2}$ & {$[4.25,4.26]$} \\
\hline 1 & $E$ & {$[3.41,4.26]$} \\
\hline 2 & $A_{1}$ & {$[2.50,4.44]$} \\
\hline 2 & $A_{2}$ & {$[3.67,3.67]$} \\
\hline 2 & $B_{1}$ & {$[3.60,3.60]$} \\
\hline 2 & $B_{2}$ & {$[4.39,4.39]$} \\
\hline 3 & $A_{1}$ & {$[2.65,4.66]$} \\
\hline 3 & $E$ & {$[2.94,4.68]$} \\
\hline 4 & $A_{1}$ & {$[2.02,3.28]$} \\
\hline 4 & $B_{1}$ & {$[3.21,3.21]$} \\
\hline \multicolumn{3}{|c|}{ (f) $\mathrm{cA} 2.60 .32$, three pions } \\
\hline 0 & $A_{1}^{-}$ & {$[3.06,4.40]$} \\
\hline 0 & $E^{-}$ & {$[4.31,4.31]$} \\
\hline 1 & $A_{2}$ & {$[3.50,4.86]$} \\
\hline 1 & $B_{2}$ & {$[4.56,4.74]$} \\
\hline 1 & $E$ & {$[4.56,4.56]$} \\
\hline 2 & $A_{1}$ & {$[4.84,5.01]$} \\
\hline 2 & $A_{2}$ & {$[3.72,5.23]$} \\
\hline 2 & $B_{1}$ & {$[4.98,5.13]$} \\
\hline 2 & $B_{2}$ & {$[4.79,5.06]$} \\
\hline 3 & $A_{1}$ & {$[5.32,5.32]$} \\
\hline 3 & $A_{2}$ & {$[3.94,5.58]$} \\
\hline 3 & $E$ & {$[4.21,5.42]$} \\
\hline 4 & $A_{2}$ & {$[3.51,4.86]$} \\
\hline 4 & $B_{2}$ & {$[4.51,4.71]$} \\
\hline 4 & $E$ & {$[4.70,4.70]$} \\
\hline
\end{tabular}

\section{B.1 General technicalities}

In both, the two and three-particle sector, we define the $\chi^{2}$ as:

$$
\chi^{2}=\sum_{i j}\left(E_{i}^{\text {data }}-E_{i}^{\text {predicted }}\right)(C)_{i j}^{-1}\left(E_{i}^{\text {data }}-E_{j}^{\text {predicted }}\right)
$$

where $C$ is the covariance matrix of the energy levels, estimated from the bootstrap samples. Best fit parameters are obtained using the Levenberg-Marquardt algorithm.

The range of validity of the quantization conditions is limited by the first inelastic threshold. This is $E^{*}=4 M_{\pi}$ $\left(5 M_{\pi}\right)$ for the two-particle (three-particle) quantization condition. We generally include levels up to that threshold, however, for the physical point ensemble (cA2.09.48), we have included levels higher up in energy. Since the $2 \pi \rightarrow 4 \pi$, and $3 \pi \rightarrow 5 \pi$ couplings are very small, we expect this to be a valid approximation. In fact, phenomenological studies set the first relevant inelasticity to be the $\rho \pi \pi$ channel ( $E^{*} \sim 8 M_{\pi}$ for physical kinematics) $[92,95,96]$.

As mentioned in the main text, we show here additional two-pion phase shift plots: Fig. 9 for cA2.30.48, and Fig. 10 for cA2.60.32. In the case of the $s$-wave phase shift, we also compare to LO ChPT. As can be seen, the ChPT prediction describes less accurately the data at heavier pion masses compare to Fig. 2.

\section{B.2 Additional discussion on three-pion fits}

First, we perform a global fit to two- and three-particle levels

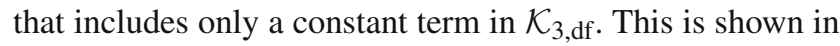
Table 10. As can be seen, the quality of the fit is significantly worse for the heavier ensembles than in the linear fits of Table 6 in the main text. For the ensemble at the physical point (cA2.09.48), the value of $\chi^{2}$ is basically the same, but in both cases $\mathcal{K}_{3 \text {,df }}$ is compatible with zero. We thus conclude that the linear model of $\mathcal{K}_{3 \text {,df }}$ in Eq. 6 in the main text is more appropriate for this system.

Next, the full covariance matrices of the fits in Table 6 in the main text are provided. We use the form $C=$ $D R D$, with $D$ being a diagonal matrix with the standard errors of the parameters. We ordered the entries as: $\left(1 / B_{0}, B_{1}, M_{\pi}^{2} \mathcal{K}_{\mathrm{df}, 3}^{\mathrm{iso}, 0}, M_{\pi}^{2} \mathcal{K}_{\mathrm{df}, 3}^{\mathrm{iso}, 1}\right)$.

cA2.09.48: $D=\operatorname{diag}(0.0086,1.1,800,500)$,

$$
R=\left(\begin{array}{cccc}
1 . & 0.73 & -0.37 & -0.02 \\
0.73 & 1 . & -0.25 & 0.11 \\
-0.37 & -0.25 & 1 . & -0.71 \\
-0.02 & 0.11 & -0.71 & 1 .
\end{array}\right),
$$

cA2.30.48: $D=\operatorname{diag}(0.015,0.4,3800,3800)$,

$$
R=\left(\begin{array}{cccc}
1.0 & 0.80 & -0.55 & 0.41 \\
0.80 & 1.0 & -0.40 & 0.35 \\
-0.55 & -0.40 & 1.0 & -0.93 \\
0.41 & 0.35 & -0.93 & 1.0
\end{array}\right)
$$




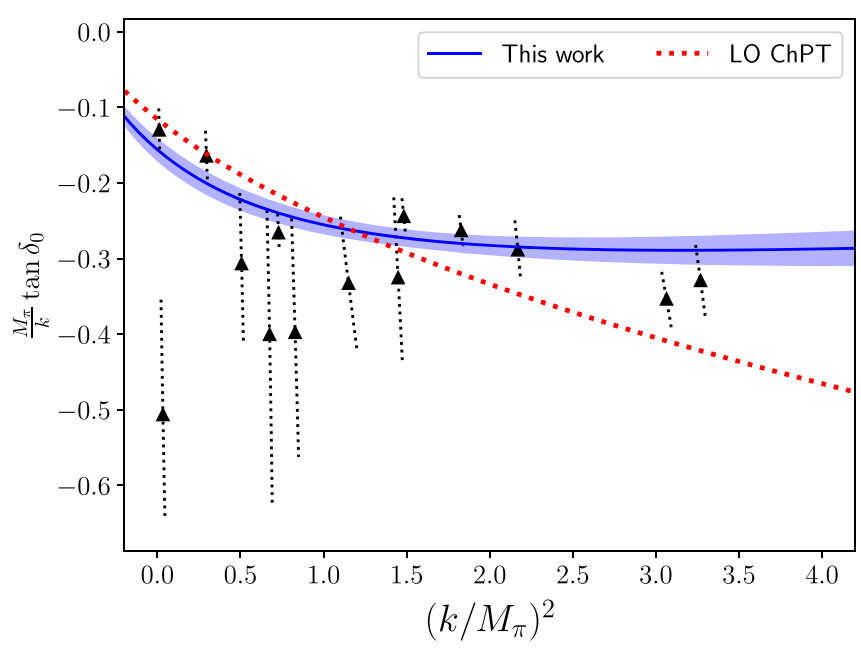

(a) $s$-wave

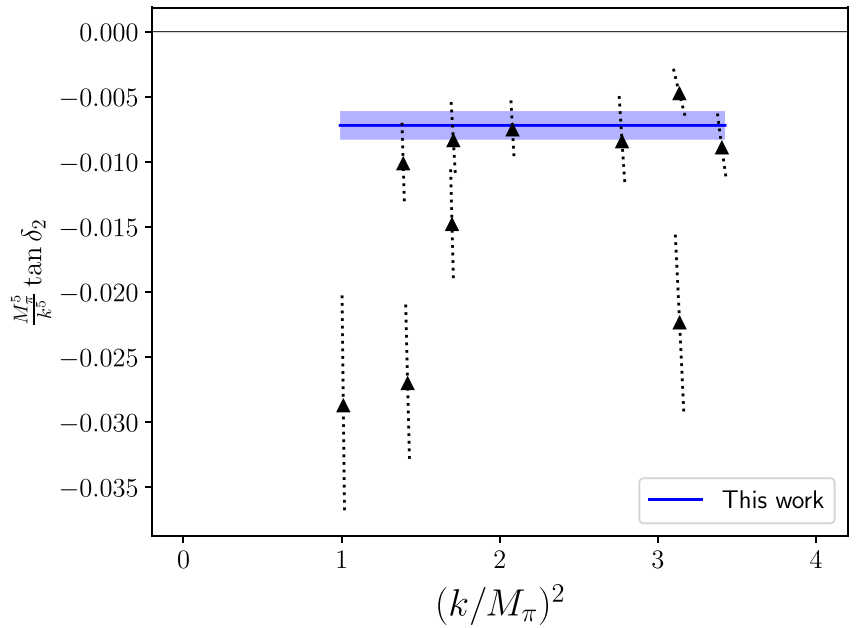

(b) $d$-wave

Fig. $9 s$ - and $d$-wave phase shift for the ensemble cA2.30.48. For $s$-wave we use a model that incorporates the Adler-zero, whereas for $d$-wave we fit to a constant in the region for which we have data. Two points have been omitted in the plot due to the very large errorbars

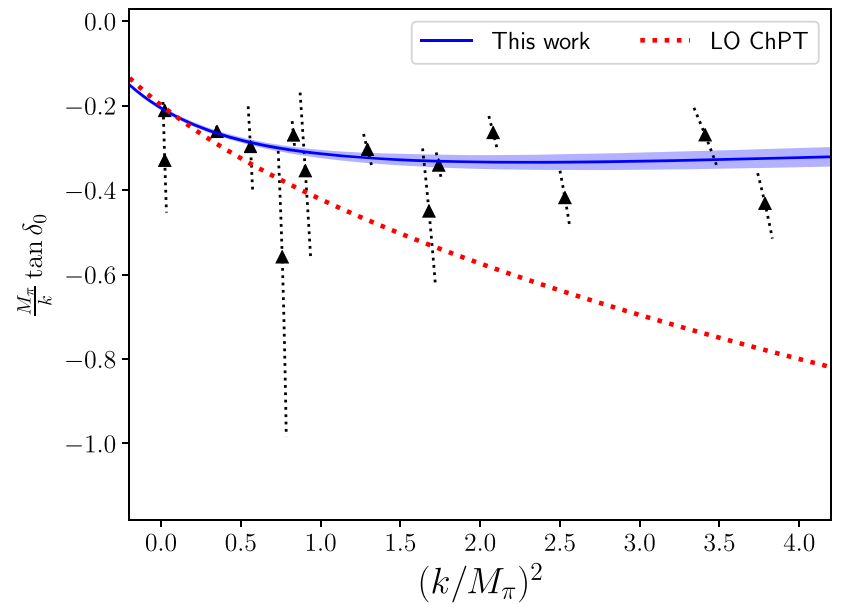

(a) $s$-wave

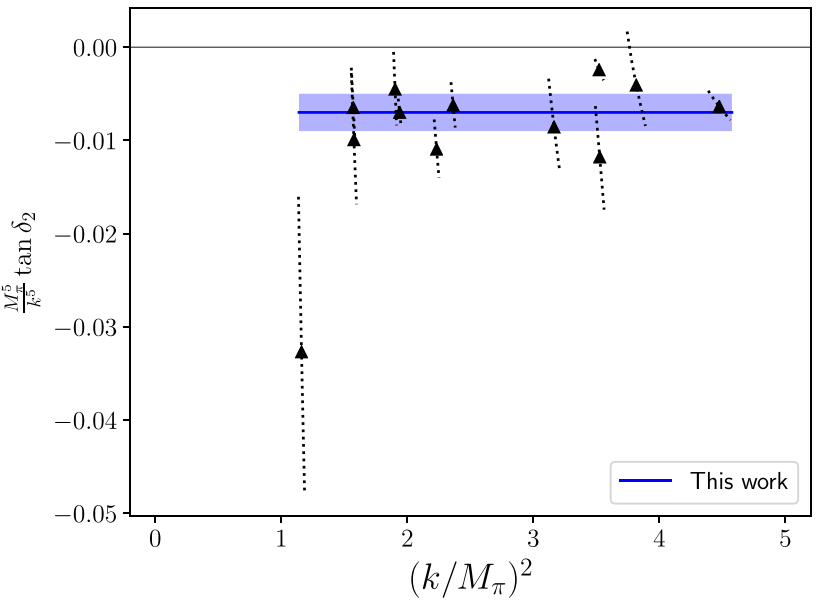

(b) $d$-wave

Fig. $10 s$-and $d$-wave phase shift for the ensemble cA2.60.32. For $s$-wave we use a model that incorporates the Adler-zero, whereas for $d$-wave we fit to a constant in the region for which we have data. Two points have been omitted in the plot due to the very large errorbars

Table 10 Two- and three-pion fits using the Adler-zero form $\left(z^{2}=M_{\pi}^{2}\right.$, fixed). Here we assume that $\mathcal{K}_{3, \mathrm{df}}$ is given by a constant: $\mathcal{K}_{3, \mathrm{df}}=\mathcal{K}_{\mathrm{df}, 3}^{\mathrm{iso}, 0}$

\begin{tabular}{llllll}
\hline & $1 / B_{0}$ & $B_{1}$ & $M_{\pi}^{2} \mathcal{K}_{\mathrm{df}, 3}^{\text {iso, } 3}$ & $M_{\pi}^{2} \mathcal{K}_{\mathrm{df}, 3}^{\text {iso, }}$ & $\chi^{2} / \mathrm{dof}$ \\
\hline cA2.60.32 & $-0.2050(49)$ & $-1.7(2)$ & $900(1000)$ & - & $71.08 /(43-3)$ \\
cA2.30.48 & $-0.149(14)$ & $-1.7(4)$ & $-2000(1400)$ & - & $47.59 /(33-3)$ \\
cA2.09.48 & $-0.0482(86)$ & $-1.3(1.1)$ & $-200(600)$ & - & $19.24 /(19-3)$ \\
\hline
\end{tabular}

cA2.60.32: $D=\operatorname{diag}(0.0049,0.2,1500,1800)$,

$$
R=\left(\begin{array}{cccc}
1.0 & 0.36 & -0.02 & 0.05 \\
0.36 & 1.0 & 0.10 & 0.22 \\
-0.02 & 0.10 & 1.0 & -0.78 \\
0.05 & 0.22 & -0.78 & 1.0
\end{array}\right) \text {, }
$$

We observe a large correlation within the two and threeparticle sectors separately - the pairs $1 / B_{0}, B_{1}$, and $M_{\pi}^{2} \mathcal{K}_{\mathrm{df}, 3}^{\mathrm{iso}, 0}, M_{\pi}^{2} \mathcal{K}_{\mathrm{df}, 3}^{\mathrm{iss}, 1}$ are highly correlated. In contrast, the correlation between the two- and three-particle sectors is milder. 


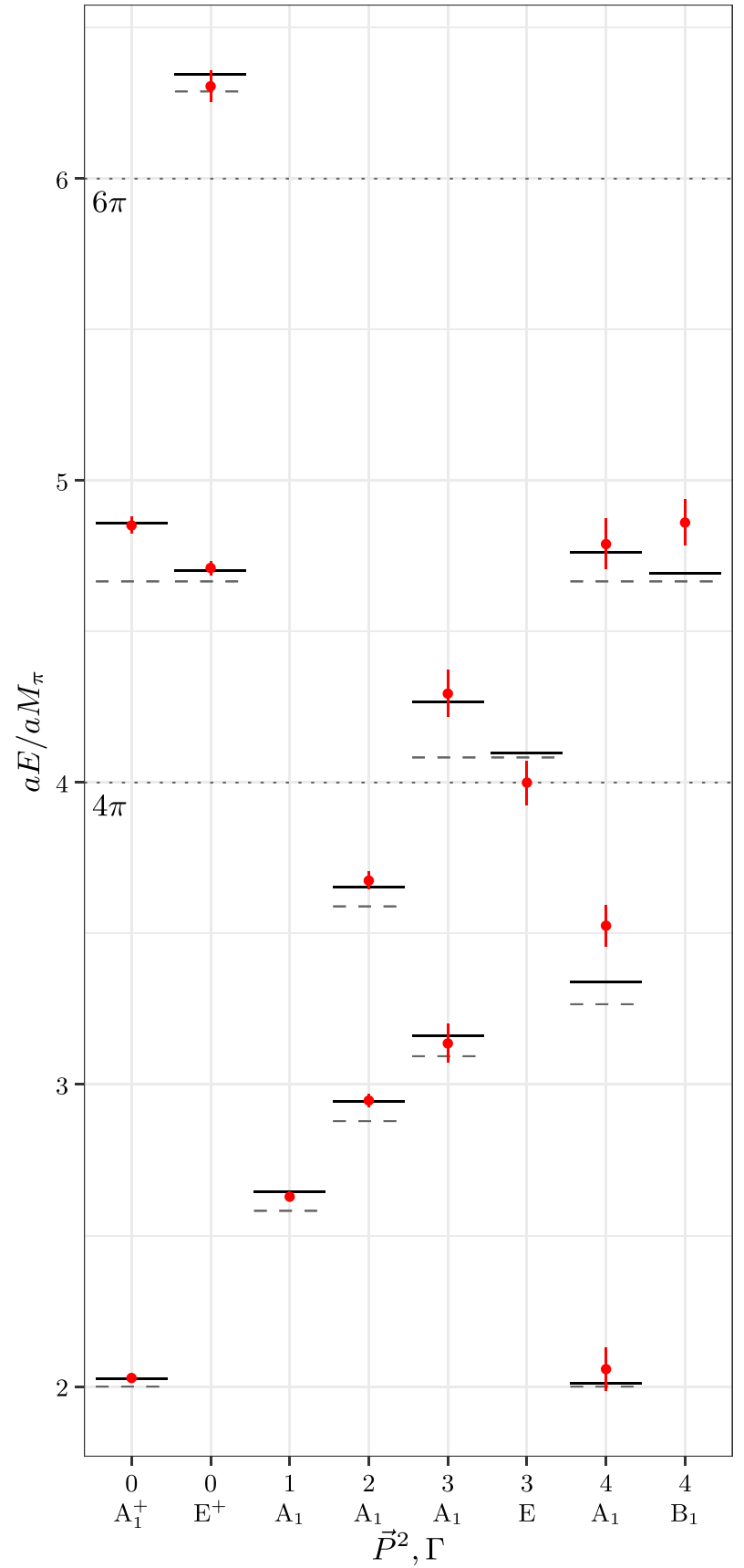

(a) Two pion channel

Fig. 11 The center-of-mass spectrum for two and three pions on the physical point ensemble (cA2.09.48). The red data points are the energy levels determined from the correlator. The black lines denote the prediction from the quantization condition. For the two-pion $A_{1}$ levels, and all three-pion levels, we use the fit in Table 6 in the main text. For the

\section{B.3 Two- and three-pion spectrum}

We conclude the discussion by comparing the spectrum from the lattice to the one predicted by the quantization conditions

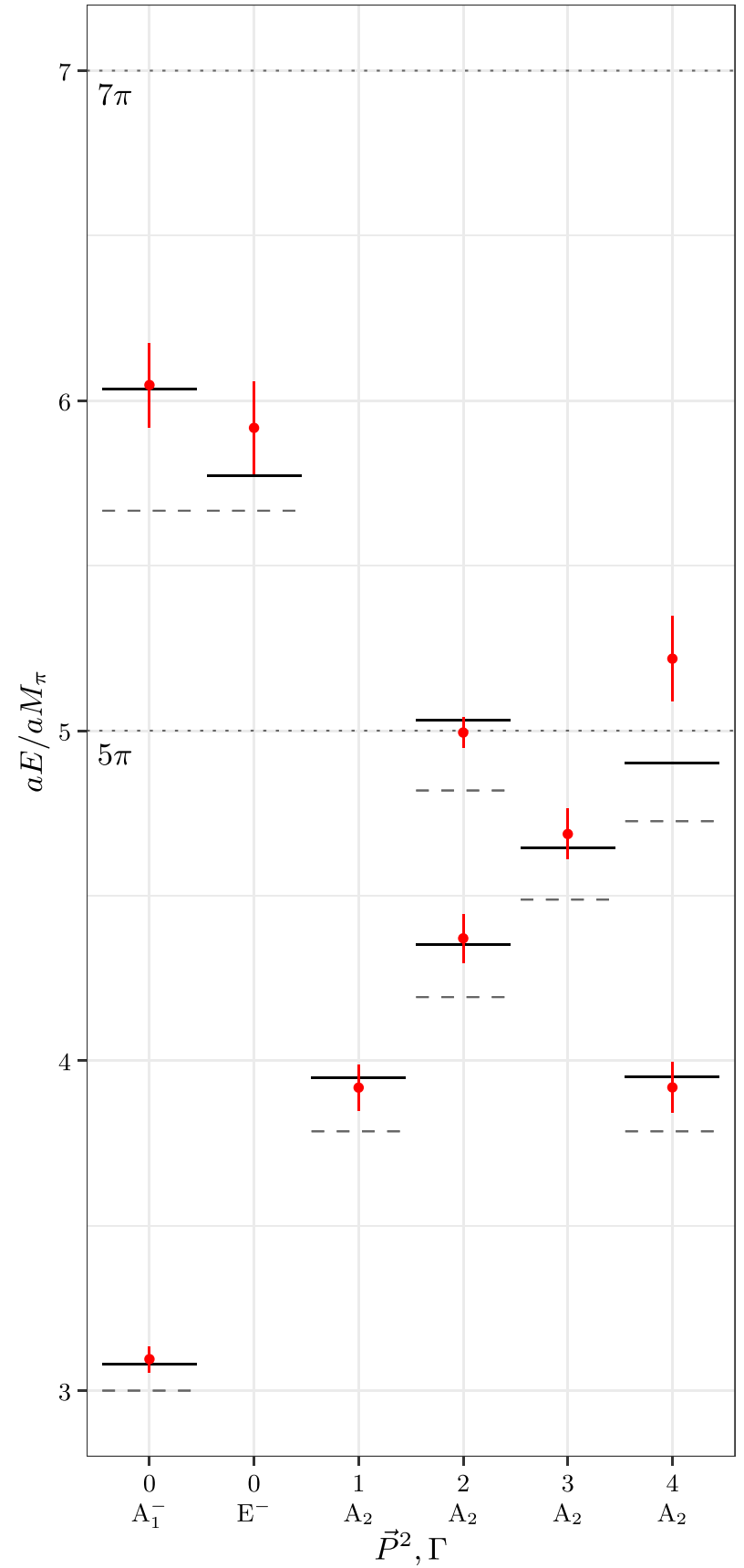

(b) Three pion channel

non- $A_{1}$ two-pion levels, which are dominated by $d$-wave interactions, we use the fit in Table 4 in the main text. The short dashed gray lines denote the noninteracting energy levels. We also include the relevant inelastic thresholds as long dotted gray lines

using the best fits. This is shown in Fig. 11 for the ensemble at the physical point. 


\section{References}

1. M. Tanabashi et al. ( PDG), Review of particle physics. Phys. Rev. D 98, 030001 (2018). https://doi.org/10.1103/PhysRevD.98. 030001

2. L. David Roper, Evidence for a $P_{11}$ pion-nucleon resonance at 556 MeV. Phys. Rev. Lett. 12, 340-342 (1964). https://doi.org/ 10.1103/PhysRevLett. 12.340

3. C. Helmes, C. Jost, B. Knippschild, C. Liu, J. Liu, L. Liu, C. Urbach, M. Ueding, Z. Wang, M. Werner (ETM), Hadron-hadron interactions from $\mathrm{N}_{f}=2+1+1$ lattice QCD: isospin-2 $\pi \pi$ scattering length. JHEP 09, 109 (2015). https://doi.org/10.1007/ JHEP09(2015)109. arXiv:1506.00408 [hep-lat]

4. M. Lüscher, Volume dependence of the energy spectrum in massive quantum field theories. 2. Scattering states. Commun. Math. Phys. 105, 153-188 (1986). https://doi.org/10.1007/BF01211097

5. M. Lüscher, Two particle states on a torus and their relation to the scattering matrix. Nucl. Phys. B 354, 531-578 (1991). https://doi. org/10.1016/0550-3213(91)90366-6

6. M. Lüscher, U. Wolff, How to calculate the elastic scattering matrix in two-dimensional quantum field theories by numerical simulation. Nucl. Phys. B 339, 222-252 (1990). https://doi.org/ 10.1016/0550-3213(90)90540-T

7. K. Rummukainen, S.A. Gottlieb, Resonance scattering phase shifts on a nonrest frame lattice. Nucl. Phys. B 450, 397-436 (1995). arXiv:hep-lat/9503028. https://doi.org/10.1016/ 0550-3213(95)00313-H

8. C.H. Kim, C.T. Sachrajda, S.R. Sharpe, Finite-volume effects for two-hadron states in moving frames. Nucl. Phys. B 727, 218-243 (2005). https://doi.org/10.1016/j.nuclphysb.2005. 08.029. arXiv:hep-lat/0507006

9. X. Song He, C.L. Feng, Two particle states and the S-matrix elements in multi-channel scattering. JHEP 07, 011 (2005). https://doi.org/10.1088/1126-6708/2005/07/011. arXiv:hep-lat/0504019

10. V. Bernard, M. Lage, U.-G. Meißner, A. Rusetsky, JHEP 1101, 019 (2011). https://doi.org/10.1007/JHEP01(2011)019. arXiv: 1010.6018 [hep-lat]

11. M.T. Hansen, S.R. Sharpe, Multiple-channel generalization of Lellouch-Luscher formula. Phys. Rev. D 86, 016007 (2012). https://doi.org/10.1103/PhysRevD.86.016007. arXiv:1204.0826 [hep-lat]

12. R.A. Briceño, Z. Davoudi, Moving multichannel systems in a finite volume with application to proton-proton fusion. Phys. Rev. D 88, 094507 (2013). https://doi.org/10.1103/PhysRevD.88. 094507. arXiv:1204.1110 [hep-lat]

13. R.A. Briceño, Two-particle multichannel systems in a finite volume with arbitrary spin. Phys. Rev. D 89, 074507 (2014). https://doi.org/10.1103/PhysRevD.89.074507. arXiv:1401.3312 [hep-lat]

14. F. Romero-López, A. Rusetsky, C. Urbach, Vector particle scattering on the lattice. Phys. Rev. D 98, 014503 (2018a). https://doi. org/10.1103/PhysRevD.98.014503. arXiv:1802.03458 [hep-lat]

15. T. Luu, M.J. Savage, Extracting scattering phase-shifts in higher partial-waves from lattice QCD calculations. Phys. Rev. D 83, 114508 (2011). https://doi.org/10.1103/PhysRevD.83.114508. arXiv: 1101.3347 [hep-lat]

16. M. Göckeler, R. Horsley, M. Lage, U.G. Meißner, P.E.L. Rakow, A. Rusetsky, G. Schierholz, J.M. Zanotti, Scattering phases for meson and baryon resonances on general moving-frame lattices. Phys. Rev. D 86, 094513 (2012). https://doi.org/10.1103/ PhysRevD.86.094513. arXiv:1206.4141 [hep-lat]

17. X. Feng, K. Jansen, D.B. Renner, The pi+ pi+ scattering length from maximally twisted mass lattice QCD. Phys. Lett. B 684,
268-274 (2010). https://doi.org/10.1016/j.physletb.2010.01.018. arXiv:0909.3255 [hep-lat]

18. M. Lage, U.-G. Meißner, A. Rusetsky, A method to measure the antikaon-nucleon scattering length in lattice QCD. Phys. Lett. B 681, 439-443 (2009). https://doi.org/10.1016/j.physletb.2009.10. 055. arXiv:0905.0069 [hep-lat]

19. D.J. Wilson, R.A. Briceño, J.J. Dudek, R.G. Edwards, C.E. Thomas, Coupled $\pi \pi, K \bar{K}$ scattering in $P$-wave and the $\rho$ resonance from lattice QCD. Phys. Rev. D 92, 094502 (2015). https:// doi.org/10.1103/PhysRevD.92.094502. arXiv:1507.02599 [hep$\mathrm{ph}]$

20. R.A. Briceño, J.J. Dudek, R.G. Edwards, D.J. Wilson, Isoscalar $\pi \pi$ scattering and the $\sigma$ meson resonance from QCD. Phys. Rev. Lett. 118, 022002 (2017). https://doi.org/10.1103/PhysRevLett. 118.022002. arXiv:1607.05900 [hep-ph]

21. R. Brett, J. Bulava, J. Fallica, A. Hanlon, B. Höz, C. Morningstar, Determination of $s$ - and $p$-wave $I=1 / 2 K \pi$ scattering amplitudes in $N_{\mathrm{f}}=2+1$ lattice QCD. Nucl. Phys. B 932, 29-51 (2018). https://doi.org/10.1016/j.nuclphysb.2018.05.008. arXiv: 1802.03100 [hep-lat]

22. C.W. Andersen, J. Bulava, B. Hörz, C. Morningstar, Elastic $I=3 / 2 p$-wave nucleon-pion scattering amplitude and the $\Delta(1232)$ resonance from $\mathrm{N}_{f}=2+1$ lattice QCD. Phys. Rev. D 97, 014506 (2018). https://doi.org/10.1103/PhysRevD.97. 014506. arXiv: 1710.01557 [hep-lat]

23. D. Guo, A. Alexandru, R. Molina, M. Mai, M. Döring, Extraction of isoscalar $\pi \pi$ phase-shifts from lattice QCD. Phys. Rev. D 98, 014507 (2018). https://doi.org/10.1103/PhysRevD.98. 014507. arXiv: 1803.02897 [hep-lat]

24. C. Andersen, J. Bulava, B. Hörz, C. Morningstar, The $I=1$ pionpion scattering amplitude and timelike pion form factor from $N_{\mathrm{f}}=$ 2+1 lattice QCD. Nucl. Phys. B 939, 145-173 (2019). https://doi. org/10.1016/j.nuclphysb.2018.12.018. arXiv:1808.05007 [heplat]

25. J.J. Dudek, R.G. Edwards, C.E. Thomas, D.J. Wilson (Hadron Spectrum), Resonances in coupled $\pi K-\eta K$ scattering from quantum chromodynamics. Phys. Rev. Lett. 113, 182001 (2014). https://doi.org/10.1103/PhysRevLett.113. 182001. arXiv:1406.4158 [hep-ph]

26. J.J. Dudek, R.G. Edwards, D.J. Wilson (Hadron Spectrum), An $a_{0}$ resonance in strongly coupled $\pi \eta, K \bar{K}$ scattering from lattice QCD. Phys. Rev. D 93, 094506 (2016). https://doi.org/10.1103/ PhysRevD.93.094506. arXiv:1602.05122 [hep-ph]

27. A. Woss, C.E. Thomas, J.J. Dudek, R.G. Edwards, D.J. Wilson, Dynamically-coupled partial-waves in $\rho \pi$ isospin-2 scattering from lattice QCD. JHEP 07, 043 (2018). https://doi.org/10.1007/ JHEP07(2018)043. arXiv:1802.05580 [hep-lat]

28. A.J. Woss, C.E. Thomas, J.J. Dudek, R.G. Edwards, D.J. Wilson, The $b_{1}$ resonance in coupled $\pi \omega, \pi \phi$ scattering from lattice QCD (2019). arXiv:1904.04136 [hep-lat]

29. C. Helmes, C. Jost, B. Knippschild, B. Kostrzewa, L. Liu, F. Pittler, C. Urbach, M. Werner (ETM), Hadron-hadron interactions from $N_{f}=2+1+1$ lattice QCD: $I=3 / 2 \pi K$ scattering length. Phys. Rev. D 98, 114511 (2018). https://doi.org/10.1103/PhysRevD.98. 114511. arXiv:1809.08886 [hep-lat]

30. L. Liu et al., Isospin- $0 \pi \pi$ s-wave scattering length from twisted mass lattice QCD. Phys. Rev. D 96, 054516 (2017). https://doi. org/10.1103/PhysRevD.96.054516. arXiv:1612.02061 [hep-lat]

31. C. Helmes, C. Jost, B. Knippschild, B. Kostrzewa, L. Liu, C. Urbach, M. Werner, Hadron-hadron interactions from $N_{f}=$ $2+1+1$ lattice QCD: isospin- $K K$ scattering length. Phys. Rev. D 96, 034510 (2017). https://doi.org/10.1103/PhysRevD.96. 034510. arXiv:1703.04737 [hep-lat]

32. M. Werner et al., Hadron-hadron interactions from $N_{f}=2+$ $1+1$ Lattice QCD: the $\rho$-resonance. Eur. Phys. J. A 56, 
61 (2020). https://doi.org/10.1140/epja/s10050-020-00057-4. arXiv:1907.01237 [hep-lat]

33. C. Culver, M. Mai, A. Alexandru, M. Döring, F.X. Lee, Pion scattering in the isospin $\mathrm{I}=2$ channel from elongated lattices (2019). arXiv:1905.10202 [hep-lat]

34. M. Mai, C. Culver, A. Alexandru, M. Döring, F.X. Lee, A cross-channel study of pion scattering from lattice QCD (2019). arXiv:1908.01847 [hep-lat]

35. M. Doring, U.G. Meißner, E. Oset, A. Rusetsky, Scalar mesons moving in a finite volume and the role of partial wave mixing. Eur. Phys. J. A 48, 114 (2012). https://doi.org/10.1140/epja/ i2012-12114-6. arXiv:1205.4838 [hep-lat]

36. M. Fischer, B. Kostrzewa, M. Mai, M. Petschlies, F. Pittler, M. Ueding, C. Urbach, M. Werner (ETM), The $\rho$-resonance with physical pion mass from $N_{f}=2$ lattice QCD (2020). arXiv:2006.13805 [hep-lat]

37. A.J. Woss, D.J. Wilson, J.J. Dudek (Hadron Spectrum), Efficient solution of the multichannel Lüscher determinant condition through eigenvalue decomposition. Phys. Rev. D 101, 114505 (2020). https://doi.org/10.1103/PhysRevD.101.114505. arXiv:2001.08474 [hep-lat]

38. J. Bulava, B. Fahy, B. Hörz, K.J. Juge, C. Morningstar, C. Him Wong, $I=1$ and $I=2 \pi-\pi$ scattering phase shifts from $N_{\mathrm{f}}=$ $2+1$ lattice QCD. Nucl. Phys. B 910, 842-867 (2016). https://doi. org/10.1016/j.nuclphysb.2016.07.024. arXiv:1604.05593 [heplat]

39. G. Rendon, L. Leskovec, S. Meinel, J. Negele, S. Paul, M. Petschlies, A. Pochinsky, G. Silvi, S. Syritsyn, $I=1 / 2 S$-wave and $P$-wave $K \pi$ scattering and the $\kappa$ and $K^{*}$ resonances from lattice QCD (2020). arXiv:2006.14035 [hep-lat]

40. C. Alexandrou, L. Leskovec, S. Meinel, J. Negele, S. Paul, M. Petschlies, A. Pochinsky, G. Rendon, S. Syritsyn, $P$-wave $\pi \pi$ scattering and the $\rho$ resonance from lattice QCD. Phys. Rev. D 96, 034525 (2017). https://doi.org/10.1103/PhysRevD.96. 034525. arXiv:1704.05439 [hep-lat]

41. R.A. Briceño, J.J. Dudek, R.D. Young, Scattering processes and resonances from lattice QCD. Rev. Mod. Phys. 90, 025001 (2018). https://doi.org/10.1103/RevModPhys.90. 025001. arXiv:1706.06223 [hep-lat]

42. M.T. Hansen, S.R. Sharpe, Relativistic, model-independent, three-particle quantization condition. Phys. Rev. D 90, 116003 (2014). https://doi.org/10.1103/PhysRevD.90.116003. arXiv: 1408.5933 [hep-lat]

43. M.T. Hansen, S.R. Sharpe, Expressing the three-particle finitevolume spectrum in terms of the three-to-three scattering amplitude. Phys. Rev. D 92, 114509 (2015). https://doi.org/10.1103/ PhysRevD.92.114509. arXiv:1504.04248 [hep-lat]

44. M.T. Hansen, S.R. Sharpe, Perturbative results for two and three particle threshold energies in finite volume. Phys. Rev. D 93, 014506 (2016a). https://doi.org/10.1103/PhysRevD.93.014506. arXiv:1509.07929 [hep-lat]

45. M.T. Hansen, S.R. Sharpe, Threshold expansion of the threeparticle quantization condition. Phys. Rev. D 93, 096006 (2016). [Erratum: Phys. Rev. D 96, 039901 (2017)]. https://doi.org/10. 1103/PhysRevD.93.096006. arXiv:1602.00324 [hep-lat]

46. R.A. Briceño, M.T. Hansen, S.R. Sharpe, Relating the finitevolume spectrum and the two-and-three-particle $S$ matrix for relativistic systems of identical scalar particles. Phys. Rev. D 95, 074510 (2017). https://doi.org/10.1103/PhysRevD.95.074510. arXiv:1701.07465 [hep-lat]

47. R.A. Briceño, M.T. Hansen, S.R. Sharpe, Numerical study of the relativistic three-body quantization condition in the isotropic approximation. Phys. Rev. D 98, 014506 (2018). https://doi.org/ 10.1103/PhysRevD.98.014506. arXiv:1803.04169 [hep-lat]

48. R.A. Briceño, M.T. Hansen, S.R. Sharpe, Three-particle systems with resonant subprocesses in a finite volume. Phys.
Rev. D 99, 014516 (2019). https://doi.org/10.1103/PhysRevD.99. 014516. arXiv:1810.01429 [hep-lat]

49. T.D. Blanton, F. Romero-López, S.R. Sharpe, Implementing the three-particle quantization condition including higher partial waves. JHEP 03, 106 (2019). https://doi.org/10.1007/ JHEP03(2019)106. arXiv:1901.07095 [hep-lat]

50. F. Romero-López, S.R. Sharpe, T.D. Blanton, R.A. Briceño, M.T. Hansen, Numerical exploration of three relativistic particles in a finite volume including two-particle resonances and bound states. JHEP 10, 007 (2019). https://doi.org/10.1007/JHEP10(2019)007. arXiv:1908.02411 [hep-lat]

51. M.T. Hansen, F. Romero-López, S.R. Sharpe, Generalizing the relativistic quantization condition to include all three-pion isospin channels. JHEP 20, 047 (2020). https://doi.org/10.1007/ JHEP07(2020)047. arXiv:2003.10974 [hep-lat]

52. T.D. Blanton, S.R. Sharpe, Equivalence of relativistic threeparticle quantization conditions (2020). arXiv:2007.16190 [heplat]

53. T.D. Blanton, S.R. Sharpe, Alternative derivation of the relativistic, three-particle quantization condition (2020). arXiv:2007.16188 [hep-lat]

54. K. Polejaeva, A. Rusetsky, Three particles in a finite volume. Eur. Phys. J. A 48, 67 (2012). https://doi.org/10.1140/epja/ i2012-12067-8. arXiv:1203.1241 [hep-lat]

55. U.-G. Meißner, G. Ríos, A. Rusetsky, Spectrum of three-body bound states in a finite volume. Phys. Rev. Lett. 114, 091602 (2015). [Erratum: Phys. Rev. Lett. 117(6), 069902 (2016)]. https:// doi.org/10.1103/PhysRevLett.117.069902. https://doi.org/10. 1103/PhysRevLett.114.091602. arXiv:1412.4969 [hep-lat]

56. H.-W. Hammer, J.-Y. Pang, A. Rusetsky, Three-particle quantization condition in a finite volume: 1 . The role of the threeparticle force. JHEP 09, 109 (2017a). https://doi.org/10.1007/ JHEP09(2017)109. arXiv:1706.07700 [hep-lat]

57. H.W. Hammer, J.Y. Pang, A. Rusetsky, Three particle quantization condition in a finite volume: 2 . general formalism and the analysis of data. JHEP 10, 115 (2017b). https://doi.org/10.1007/ JHEP10(2017)115. arXiv:1707.02176 [hep-lat]

58. M. Döring, H.W. Hammer, M. Mai, J.Y. Pang, A. Rusetsky, J. $\mathrm{Wu}$, Three-body spectrum in a finite volume: the role of cubic symmetry. Phys. Rev. D 97, 114508 (2018). https://doi.org/10. 1103/PhysRevD.97.114508. arXiv:1802.03362 [hep-lat]

59. J.-Y. Pang, J.-J. Wu, H.W. Hammer, U.-G. Meißner, A. Rusetsky, Energy shift of the three-particle system in a finite volume. Phys. Rev. D 99, 074513 (2019). https://doi.org/10.1103/PhysRevD.99. 074513. arXiv:1902.01111 [hep-lat]

60. M. Mai, M. Döring, Three-body unitarity in the finite volume. Eur. Phys. J. A 53, 240 (2017). https://doi.org/10.1140/epja/ i2017-12440-1. arXiv:1709.08222 [hep-lat]

61. M. Mai, M. Döring, Finite-volume spectrum of $\pi^{+} \pi^{+}$and $\pi^{+} \pi^{+} \pi^{+}$systems. Phys. Rev. Lett. 122, 062503 (2019). https:// doi.org/10.1103/PhysRevLett.122.062503. arXiv:1807.04746 [hep-lat]

62. P. Klos, S. König, H.W. Hammer, J.E. Lynn, A. Schwenk, Signatures of few-body resonances in finite volume. Phys. Rev. C98, 034004 (2018). https://doi.org/10.1103/PhysRevC.98. 034004. arXiv:1805.02029 [nucl-th]

63. P. Guo, V. Gasparian, An solvable three-body model in finite volume. Phys. Lett. B 774, 441-445 (2017). https://doi.org/10.1016/ j.physletb.2017.10.009. arXiv:1701.00438 [hep-lat]

64. A. Jackura, C. Fernández-Ramírez, V. Mathieu, M. Mikhasenko, J. Nys, A. Pilloni, K. Saldaña, N. Sherrill, A.P. Szczepaniak (JPAC), Phenomenology of relativistic $\mathbf{3} \rightarrow \mathbf{3}$ reaction amplitudes within the isobar approximation. Eur. Phys. J. C 79, 56 (2019). https://doi.org/10.1140/epjc/s10052-019-6566-1. arXiv:1809.10523 [hep-ph] 
65. M.T. Hansen, S.R. Sharpe, Lattice QCD and three-particle decays of resonances (2019). arXiv:1901.00483 [hep-lat]

66. Ben Hörz, Andrew Hanlon, "Two- and three-pion finite-volume spectra at maximal isospin from lattice QCD". Phys. Rev. Lett. 123, 142002 (2019). https://doi.org/10.1103/PhysRevLett.123. 142002. arXiv: 1905.04277 [hep-lat]

67. C. Culver, M. Mai, R. Brett, A. Alexandru, M. Döring, Three body spectrum from lattice QCD. Phys. Rev. D 101, 114507 (2020). https://doi.org/10.1103/PhysRevD.101.114507. arXiv:1911.09047 [hep-lat]

68. M. Mai, M. Döring, C. Culver, A. Alexandru, Three-body unitarity versus finite-volume $\pi^{+} \pi^{+} \pi^{+}$spectrum from lattice QCD. Phys. Rev. D 101, 054510 (2020). https://doi.org/10.1103/PhysRevD. 101.054510. arXiv:1909.05749 [hep-lat]

69. T.D. Blanton, F. Romero-López, S.R. Sharpe, $I=3$ threepion scattering amplitude from lattice QCD. Phys. Rev. Lett. 124, 032001 (2020). https://doi.org/10.1103/PhysRevLett.124. 032001. arXiv:1909.02973 [hep-lat]

70. P. Guo, B. Long, Multi- $\pi^{+}$systems in a finite volume. Phys. Rev. D 101, 094510 (2020). https://doi.org/10.1103/PhysRevD. 101.094510. arXiv:2002.09266 [hep-lat]

71. P. Guo, Modeling few-body resonances in finite volume (2020). arXiv:2007.12790 [hep-lat]

72. S.R. Beane, W. Detmold, M.J. Savage, n-Boson energies at finite volume and three-Boson interactions. Phys. Rev. D 76, 074507 (2007). https://doi.org/10.1103/PhysRevD.76.074507. arXiv:0707.1670 [hep-lat]

73. W. Detmold, M.J. Savage, A. Torok, S.R. Beane, T.C. Luu, K. Orginos, A. Parreno, Multi-pion states in lattice QCD and the charged-pion condensate. Phys. Rev. D 78, 014507 (2008). https://doi.org/10.1103/PhysRevD.78.014507. arXiv:0803.2728 [hep-lat]

74. F. Romero-López, A. Rusetsky, C. Urbach, Two- and threebody interactions in $\varphi^{4}$ theory from lattice simulations. Eur. Phys. J. C 78, 846 (2018b). https://doi.org/10.1140/epjc/ s10052-018-6325-8. arXiv:1806.02367 [hep-lat]

75. S.R. Beane et al., Charged multi-hadron systems in lattice QCD+QED (2020). arXiv:2003.12130 [hep-lat]

76. J.J. Dudek, R.G. Edwards, C.E. Thomas, S and D-wave phase shifts in isospin-2 pi pi scattering from lattice QCD. Phys. Rev. D 86, 034031 (2012). https://doi.org/10.1103/PhysRevD.86. 034031. arXiv:1203.6041 [hep-ph]

77. J. Gasser, H. Leutwyler, Light quarks at low temperatures. Phys. Lett. B 184, 83-88 (1987a). https://doi.org/10.1016/ 0370-2693(87)90492-8

78. J. Gasser, H. Leutwyler, Thermodynamics of chiral symmetry. Phys. Lett. B 188, 477-481 (1987b). https://doi.org/10.1016/ 0370-2693(87)91652-2

79. J. Gasser, H. Leutwyler, Spontaneously broken symmetries: effective Lagrangians at finite volume. Nucl. Phys. B 307, 763-778 (1988). https://doi.org/10.1016/0550-3213(88)90107-1

80. A. Abdel-Rehim et al., (ETM), First physics results at the physical pion mass from $N_{f}=2$ Wilson twisted mass fermions at maximal twist. Phys. Rev. D 95, 094515 (2017). https://doi.org/10.1103/ PhysRevD.95.094515 arXiv:1507.05068 [hep-lat]

81. Y. Iwasaki, Renormalization group analysis of lattice theories and improved lattice action: two-dimensional nonlinear $\mathrm{O}(\mathrm{N})$ sigma model. Nucl. Phys. B 258, 141-156 (1985). https://doi.org/10. 1016/0550-3213(85)90606-6

82. R. Frezzotti, P.A. Grassi, S. Sint, P. Weisz (Alpha), Lattice QCD with a chirally twisted mass term. JHEP 08, 058 (2001). arXiv:hep-lat/0101001

83. R. Frezzotti, G.C. Rossi, Chirally improving Wilson fermions. I: O(a) improvement. JHEP 08, 007 (2004). arXiv:hep-lat/0306014

84. M.I. Buchoff, J.-W. Chen, A. Walker-Loud, pi-pi scattering in twisted mass chiral perturbation theory. Phys. Rev. D 79,
074503 (2009). https://doi.org/10.1103/PhysRevD.79.074503. arXiv:0810.2464 [hep-lat]

85. M. Peardon, J. Bulava, J. Foley, C. Morningstar, J. Dudek, R.G. Edwards, B. Joo, H.-W. Lin, D.G. Richards, K.J. Juge (Hadron Spectrum), A novel quark-field creation operator construction for hadronic physics in lattice QCD. Phys. Rev. D 80, 054506 (2009). https://doi.org/10.1103/PhysRevD.80.054506. arXiv:0905.2160 [hep-lat]

86. C. Morningstar, J. Bulava, J. Foley, K.J. Juge, D. Lenkner, M. Peardon, C.H. Wong, Improved stochastic estimation of quark propagation with Laplacian Heaviside smearing in lattice QCD. Phys. Rev. D 83, 114505 (2011). https://doi.org/10.1103/PhysRevD.83. 114505. arXiv:1104.3870 [hep-lat]

87. P. Dimopoulos et al., Topological susceptibility and $\eta^{\prime}$ meson mass from $N_{f}=2$ lattice QCD at the physical point. Phys. Rev. D 99, 034511 (2019). https://doi.org/10.1103/PhysRevD.99. 034511. arXiv:1812.08787 [hep-lat]

88. C. Michael, I. Teasdale, Extracting glueball masses from lattice QCD. Nucl. Phys. B215, 433-446 (1983). https://doi.org/ 10.1016/0550-3213(83)90674-0

89. B. Blossier, M.D. Morte, G. von Hippel, T. Mendes, R. Sommer, On the generalized eigenvalue method for energies and matrix elements in lattice field theory. JHEP 04, 094 (2009). https://doi. org/10.1088/1126-6708/2009/04/094. arXiv:0902.1265 [hep-lat]

90. M. Fischer, B. Kostrzewa, J. Ostmeyer, K. Ottnad, M. Ueding, C. Urbach, On the generalised eigenvalue method and its relation to Prony and generalised pencil of function methods (2020b). arXiv:2004.10472 [hep-lat]

91. M. Ueding, $N_{\mathrm{f}}=2$ three pion $I=3$ scattering data repository. https://github.com/HISKP-LQCD/Nf2-3pi-I3-scattering-data

92. R. Kaminski, J.R. Pelaez, F.J. Yndurain, The Pion-pion scattering amplitude. III. Improving the analysis with forward dispersion relations and Roy equations. Phys. Rev. D 77, 054015 (2008). https://doi.org/10.1103/PhysRevD.77.054015. arXiv:0710.1150 [hep-ph]

93. G. Colangelo, J. Gasser, H. Leutwyler, $\pi \pi$ scattering. Nucl. Phys. B 603, 125-179 (2001). https://doi.org/10.1016/ S0550-3213(01)00147-X. arXiv:hep-ph/0103088

94. S.L. Adler, Consistency conditions on the strong interactions implied by a partially conserved axial-vector current. Phys. Rev. 137, B1022-B1033 (1965). https://doi.org/10.1103/PhysRev. 137.B1022

95. F.J. Yndurain, Low-energy pion physics (2002). arXiv:hep-ph/0212282

96. J.R. Pelaez, F.J. Yndurain, The Pion-pion scattering amplitude. Phys. Rev. D 71, 074016 (2005). https://doi.org/10.1103/ PhysRevD.71.074016. arXiv:hep-ph/0411334

97. I. Caprini, G. Colangelo, H. Leutwyler, Regge analysis of the pi pi scattering amplitude. Eur. Phys. J. C 72, 1860 (2012). https://doi. org/10.1140/epjc/s10052-012-1860-1. arXiv:1111.7160 [hep$\mathrm{ph}]$

98. M. Albaladejo, J.A. Oller, On the size of the sigma meson and its nature. Phys. Rev. D 86, 034003 (2012). https://doi.org/10.1103/ PhysRevD.86.034003. arXiv:1205.6606 [hep-ph]

99. T. Yamazaki et al. (CP-PACS), $I=2$ pi pi scattering phase shift with two flavors of $\mathrm{O}$ (a) improved dynamical quarks. Phys. Rev. D 70, 074513 (2004). https://doi.org/10.1103/PhysRevD.70. 074513. arXiv:hep-lat/0402025

100. S.R. Beane, P.F. Bedaque, K. Orginos, M.J. Savage (NPLQCD), $\mathrm{I}=2$ pi-pi scattering from fully-dynamical mixed-action lattice QCD. Phys. Rev. D 73, 054503 (2006). https://doi.org/10.1103/ PhysRevD.73.054503. arXiv:hep-lat/0506013

101. S.R. Beane, T.C. Luu, K. Orginos, A. Parreno, M.J. Savage, A. Torok, A. Walker-Loud, Precise determination of the $\mathrm{I}=2$ pi pi scattering length from mixed-action lattice QCD. Phys. 
Rev. D 77, 014505 (2008). https://doi.org/10.1103/PhysRevD.77. 014505. arXiv:0706.3026 [hep-lat]

102. S.R. Beane, E. Chang, W. Detmold, H.W. Lin, T.C. Luu, K. Orginos, A. Parreno, M.J. Savage, A. Torok, A. Walker-Loud (NPLQCD), The I=2 pipi S-wave Scattering Phase Shift from Lattice QCD. Phys. Rev. D 85, 034505 (2012). https://doi.org/ 10.1103/PhysRevD.85.034505. arXiv:1107.5023 [hep-lat]

103. T. Yagi, S. Hashimoto, O. Morimatsu, M. Ohtani, $\mathrm{I}=2 \pi-$ $\pi$ scattering length with dynamical overlap fermion (2011). arXiv:1108.2970 [hep-lat]

104. F. Ziwen, Lattice QCD study of the s-wave $\pi \pi$ scattering lengths in the $\mathrm{I}=0$ and 2 channels. Phys. Rev. D 87, 074501 (2013). https://doi.org/10.1103/PhysRevD.87.074501. arXiv:1303.0517 [hep-lat]

105. K. Sasaki, N. Ishizuka, M. Oka, T. Yamazaki (PACS-CS), Scattering lengths for two pseudoscalar meson systems. Phys. Rev. D 89, 054502 (2014). https://doi.org/10.1103/PhysRevD.89. 054502. arXiv:1311.7226 [hep-lat]

106. J. Bijnens, G. Colangelo, G. Ecker, J. Gasser, M.E. Sainio, "Pionpion scattering at low energy," Nucl. Phys. B 508, 263-310 ( 1997), [Erratum: Nucl.Phys.B 517, 639-639 (1998)], https://doi. org/10.1016/S0550-3213(97)00621-4, arXiv:hep-ph/9707291

107. Jülich Supercomputing Centre, JUQUEEN: IBM Blue Gene/Q Supercomputer System at the Jülich Supercomputing Centre. J. Large-Scale Res. Facil. 1 (2015). https://doi.org/10.17815/ jlstr-1-18

108. Jülich Supercomputing Centre, JURECA: modular supercomputer at Jülich Supercomputing Centre. J. Large-Scale Res. Facil. 4 (2018). https://doi.org/10.17815/jlsrf-4-121-1

109. Jülich Supercomputing Centre, JUWELS: Modular Tier-0/1 Supercomputer at the Jülich Supercomputing Centre. J. LargeScale Res. Facil. 5 (2019). https://doi.org/10.17815/jlsrf-5-171

110. K. Jansen, C. Urbach, tmLQCD: a program suite to simulate Wilson Twisted mass lattice QCD. Comput. Phys. Commun. 180, 2717-2738 (2009). https://doi.org/10.1016/j.cpc.2009.05. 016. arXiv:0905.3331 [hep-lat]

111. A. Abdel-Rehim, F. Burger, A. Deuzeman, K. Jansen, B. Kostrzewa, L. Scorzato, C. Urbach, Recent developments in the tmLQCD software suite. PoS LATTICE2013, 414 (2014). https://doi.org/10.22323/1.187.0414. arXiv:1311.5495 [hep-lat]
112. A. Deuzeman, K. Jansen, B. Kostrzewa, C. Urbach, Experiences with OpenMP in tmLQCD. PoS LATTICE2013, 416 (2013). arXiv: 1311.4521 [hep-lat]

113. A. Deuzeman, S. Reker, C. Urbach (ETM), Lemon: an MPI parallel I/O library for data encapsulation using LIME. Comput. Phys. Commun. 183, 1321-1335 (2012). https://doi.org/10.1016/j.cpc. 2012.01.016. arXiv:1106.4177 [hep-lat]

114. M.A. Clark, R. Babich, K. Barros, R.C. Brower, C. Rebbi, Solving Lattice QCD systems of equations using mixed precision solvers on GPUs. Comput. Phys. Commun. 181, 1517-1528 (2010). https://doi.org/10.1016/j.cpc.2010.05.002. arXiv:0911.3191 [hep-lat]

115. R. Babich, M.A. Clark, B. Joo, G. Shi, R.C. Brower, S. Gottlieb, Scaling lattice QCD beyond 100 GPUs, in SC11 International Conference for High Performance Computing, Networking, Storage and Analysis Seattle, Washington, November 12-18, 2011 (2011). https://doi.org/10.1145/2063384.2063478. arXiv:1109.2935 [hep-lat]

116. M.A. Clark, B. Joó, A. Strelchenko, M. Cheng, A. Gambhir, R. Brower, Accelerating lattice QCD multigrid on GPUs using finegrained parallelization (2016). arXiv:1612.07873 [hep-lat]

117. R Core Team, R: a language and environment for statistical computing. R Foundation for Statistical Computing, Vienna (2019). https://www.R-project.org/

118. B. Kostrzewa, J. Ostmeyer, M. Ueding, C. Urbach, hadron: package to extract hadronic quantities (2020). R package version 3.0.1. https://github.com/HISKP-LQCD/hadron

119. M. Ueding, paramvalf: parameter value analysis framework (b), $r$ package version 2.7.0

120. U. Wolff (ALPHA), Monte Carlo errors with less errors. Comput. Phys. Commun. 156, 143-153 (2004). https://doi.org/10.1016/ S0010-4655(03)00467-3. https://doi.org/10.1016/j.cpc.2006.12. 001. [Erratum: Comput. Phys. Commun. 176, 383 (2007)]. arXiv:hep-lat/0306017 [hep-lat] 